\title{
Improvement of k-epsilon Turbulence Model for CFD Simulation of Atmospheric Boundary Layer around a High-rise Building Using Stochastic Optimization and Monte Carlo Sampling Technique
}

\author{
Mohammadreza Shirzadi ${ }^{a}$, Parham A. Mirzaei ${ }^{\text {b,1, }}$, Mohammad Naghashzadegana \\ ${ }^{\text {a }}$ Engineering Department, University of Guilan, Rasht, Iran \\ ${ }^{\mathrm{b}}$ Architecture and Built Environment Department, University of Nottingham, Nottingham, UK
}

\begin{abstract}
The accuracy of the computational fluid dynamics (CFD) to model the airflow around the buildings in the atmospheric boundary layer (ABL) is directly linked to the utilized turbulence model. Despite the popularity and their low computational cost, the current Reynolds Averaged Navier-Stokes (RANS) models cannot accurately resolve the wake regions behind the buildings. The default values of the RANS models' closure coefficients in CFD tools such as ANSYS CFX, ANSYS FLUENT, PHOENIX, and STAR CCM+ are mainly adapted from other fields and physical problems, which are not perfectly suitable for ABL flow modeling. This study embarks on proposing a systematic approach to find the optimum values for the closure coefficients of RANS models in order to significantly improve the accuracy of CFD simulations for urban studies. The methodology is based on stochastic optimization and Monte Carlo Sampling technique. To show the capability of the method, a test case of airflow around an isolated building placed in a non-isothermal unstable ABL was considered. The recommended values for this case study in accordance with the optimization method were thus found to be $1.45 \leq C_{\varepsilon 1} \leq 1.5$, of $2.7 \leq C_{\varepsilon 2} \leq 3$, and $0.12 \leq C_{\mu} \leq 0.15$. The default value of $\sigma_{k}=1$ is suggested to be acceptable while the value of $\sigma_{\varepsilon}$ is obtained through a correlation. The error of the estimated reattachment length behind the building decreased form $170 \%$ for the default values to $28 \%$ for the modified values.
\end{abstract}

Keywords: CFD, Turbulence, Optimization, Microclimate, Monte Carlo Sampling, Atmospheric Boundary Layer

\footnotetext{
${ }^{1}$ Corresponding author: University Park, Nottingham, NG2RD, UK

Tel.: +44 011595 14129; fax: +44 01159513159

Email: Parham.Mirzaei_Ahranjani@nottingham.ac.uk
} 


\begin{tabular}{|clcl}
\hline$\rho$ & Density & $U_{H}$ & Inflow mean streamwise velocity at \\
& & & building height $H$ \\
$t$ & Time & $H$ & Building height \\
$x_{i}$ & Component of space coordinate & $\alpha$ & Power-law exponent \\
$U_{i}$ & Component of the mean velocity vector & $q$ & Hit rate \\
$\tau_{i j}$ & Viscous stress tensor & $N$ & Number of data points (48) \\
$S_{M i}$ & Body forces & $O_{i}$ & Observed value \\
$\mu_{t}$ & Turbulent viscosity & $P_{i}$ & Predicted value \\
$\delta_{i j}$ & Kronecker Delta function & $F A C 2$ & The fraction of the predictions within a \\
& & & factor of 2 of the observations \\
$k$ & Turbulent kinetic energy & $\boldsymbol{X}_{f}$ & Reattachment length behind the building \\
$g_{i}$ & Gravity vector & $\boldsymbol{X}_{r}$ & Reattachment length on the roof \\
$C_{\mu}$ & $k-\varepsilon$ model constant & $u_{i}$ & Fluctuating velocity component in the \\
& & & turbulent flow \\
$\mu$ & Molecular viscosity & $\sigma_{k}$ & $k-\varepsilon$ model constant \\
$\varepsilon$ & Turbulent dissipation rate & $\sigma_{\varepsilon}$ & $k-\varepsilon$ model constant \\
$P_{k}$ & Shear production term in $k$-equation & $C_{\varepsilon 2}$ & $k-\varepsilon$ model constant \\
$P_{k b}$ & Buoyant production term in $k$-equation & $\theta_{H}$ & Temperature at building height $\left(11^{\circ} \mathrm{C}\right)$ \\
$C_{\varepsilon 1}$ & $k-\varepsilon$ model constant & $\Delta \theta$ & $\theta_{f}-\theta_{H}$ \\
$\theta_{f}$ & Floor temperature $\left(45^{\circ} \mathrm{C}\right)$ & & \\
\hline
\end{tabular}

\section{Introduction and literature review}

Airflow modeling in built environment has a significant potential to help urban planners, architects and engineers in the design stages of buildings and cities (Capeluto et al, 2003; Murakami, 2006; Wong et al, 2011). In particular, an accurate modeling can bring about desired outcomes such as the improvement of the pedestrian-level wind comfort (Haghighat and Mirzaei, 2011; Mirzaei and Haghighat, 2012; Richards et al, 2002; Tsang et al, 2012), reduction of the pollution dispersion (Mirzaei and Haghighat, 2010, 2011; Yamada, 2004), minimizing the building energy consumption (Allegrini et al, 2015; Evins et al, 2014; Yi and Feng, 2013), utilizing wind energy for modern applications (Mirzaei and Rad, 2013), and mitigation of the urban heat island (Magli et al, 2015;

47 Mirzaei, 2015). Among different techniques for analyzing airflow in outdoor climates such as wind tunnel experiments and on-site measurements, Computational Fluid Dynamics (CFD) emerged as a reliable and cost effective method to simulate the wind condition around buildings. Atmospheric

50 boundary layer airflow around the buildings, as displayed in Fig.1(a), includes complex phenomena, such as separation, reattachment, large-scale turbulence and unsteady vortex shedding (Rodi, 1997); hence turbulence modeling has a significant impact on the accuracy of the CFD models. Despite many 53 years of researches, CFD modeling of turbulent flow around buildings still remains a challenging issue 54 (Lateb et al, 2016). Even for a simple cubic form of an isolated building, there is a noticeable disagreement between the experimental results and CFD predictions (see Fig.1(b) and Fig.1(c)). 


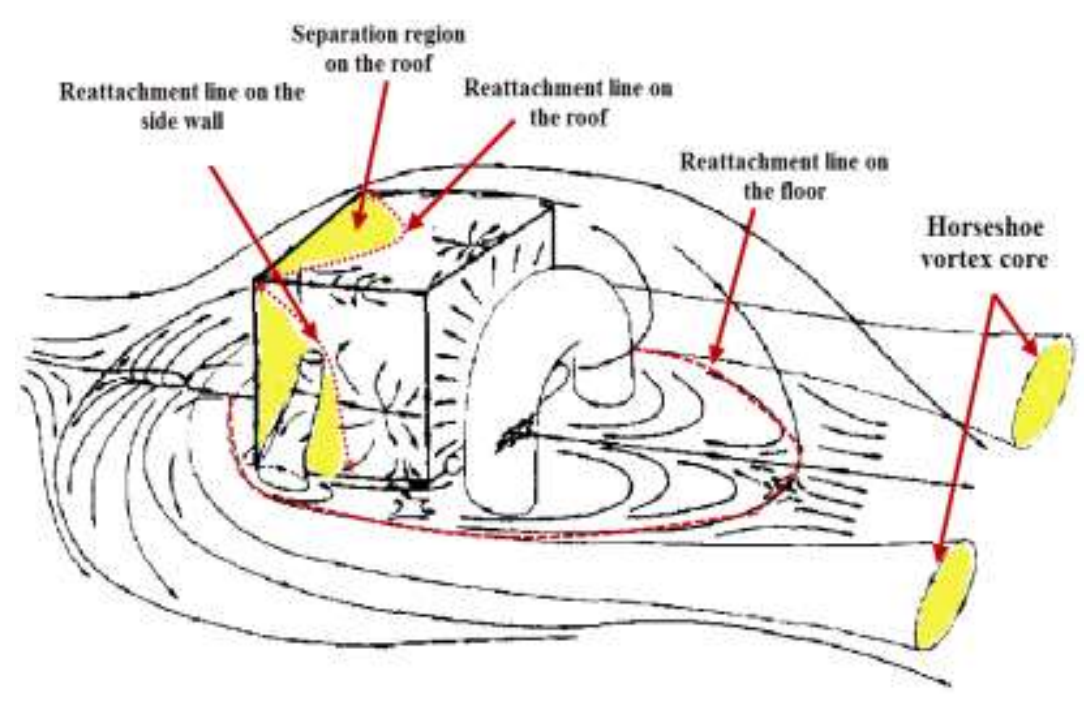

(a)

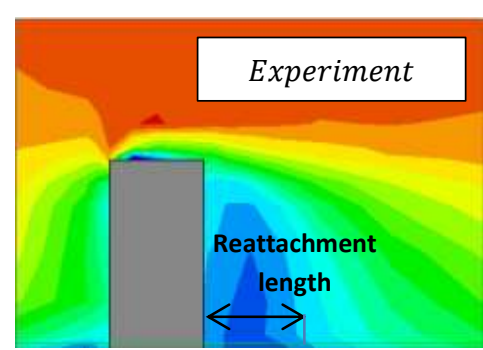

(b)

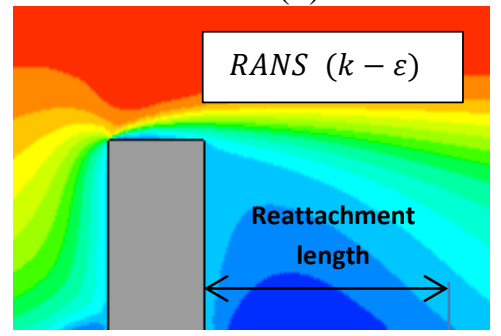

(c)

Figure 1 (a) Flow visualization around an isolated building (Hunt et al, 1978). Streamwise velocity distribution around an isolated building for (b) experiment by Yoshie et al (2011), (c) RANS turbulence mode

Early works presented in (Lakehal and Rodi, 1997; Murakami, 1993; Murakami et al, 1990; Tamura et al, 1997) examined different turbulence models to predict the airflow around a generic bluff body via focusing on the pressure distribution and separation of flow over the roof. In an attempt to investigate the problem of the airflow modeling in urban areas, a working group for CFD modeling of the wind environment around a building was organized by the Architectural Institute of Japan (Shirasawa et al, 2003). Tominaga et al (2004) presented the result of a cross comparison of the airflow around a single high-rise building in the lower part of the atmospheric boundary layer (ABL). Also, they performed numerical simulation of a building complex in an actual urban region. Different software and turbulence models were examined in their study for two test cases of 2:1:1 and 4:4:1 shaped building models based on the experiments from Yan and Kazuki (1998). Their results showed that the standard $\mathrm{k}-\varepsilon$ model mainly fails to produce the reverse flow over the roof, but revised models (e.g. LK k $-\varepsilon$ (Kato, 1993), RNG k $-\varepsilon$ (Yakhot and Orszag, 1986), MMK k $-\varepsilon$ (Tsuchiya et al, 1997) could more accurately predict the flow pattern. However, the standard $\mathrm{k}-\varepsilon$ model and all revised models overestimated the reattachment length behind the building.

A similar finding was presented by Yoshie et al (2007), Tominaga and Stathopoulos (2010), Vardoulakis et al (2011), and Gousseau et al (2011) which emphasized the inaccuracy of the Reynolds Averaged Navier Stokes (RANS) turbulence models in reproducing the weak wind regions behind buildings and also in overestimating the reattachment length behind the building. In another study by Köse and Dick (2010), it was shown that the poor accuracy of the RANS turbulence models for prediction of the airflow around the buildings in ABL is accompanied with a low accuracy in estimating the mean surface pressure over the building in comparison with LES models.

In a recent study by Tominaga (2015), the accuracy of the unsteady Reynolds-averaged NavierStokes (URANS) turbulence modeling for an isolated building was investigated. He concluded that the URANS simulation based on the $k-\omega S S T$ turbulence model is able to simulate the unsteady fluctuations behind the building and providing a better velocity field in this region as well; however, the model generally overestimates the separation in the corners. 
The previous literature clearly demonstrates that the linear two-equation RANS turbulence models provide poor results for the airflow prediction around an isolated building, compared with the URANS and LES models. However, high complexity of the URANS and LES models in specifying accurate boundary condition, proper mesh size and time scale, in addition to their inherent high computational cost keep their potential application as a reliable and fast solution for many realistic engineering problems very limited. Despite the development of several methods for improving the RANS turbulence models, e.g. $R N G k-\varepsilon$ (Yakhot et al, 1992) and Realizable $\mathrm{k}-\varepsilon$ (Shih et al, 1995), their application for the airflow modeling around buildings in ABL is limited due to their poor accuracy in resolving the flow in the weak wind regions.

Moreover, another limitation of the current RANS family models refers to their semi-empirical coefficients, which are mainly adapted from the fundamental and classical flow problems, e.g. homogenous decaying turbulence, free sheer flow, and fully developed channel flow. The value of these coefficients collected in the work carried out by Launder and Spalding (1974) are shown in Table 1. These values are used in most CFD tools such as ANSYS CFX, ANSYS FLUENT, PHOENIX, and STAR CCM+ as default parameters. However, experimental measurements performed in different studies show a slight difference in values for these coefficients. For instance, Mohamed and LaRue (1990) suggested a value of $C_{\varepsilon 2}=1.77$ which is lower than the default value of 1.92 . Experimental and numerical analyses by Kim et al (1987) demonstrate that the variation of $C_{\mu}$ for a channel flow in areas far from the wall $\left(y^{+}>50\right)$ is between 0.06 to 0.095 , resulting in an average value of $C_{\mu}=0.09$. The value of $C_{\mu}$ for a temporal-mixing layer was reported between 0.07 and 0.11 (Pope, 2001). In an experimental work by Tavoularis and Karnik (1989), different values for the ratio $\frac{C_{\varepsilon 2}-1}{C_{\varepsilon 1}-1}$, ranging from 1.33 to 1.75 , were observed for different shear flows. Once default values of $C_{\varepsilon 1}$ and $C_{\varepsilon 2}$ are used, the ratio gets 2.09 , which is noticeably different from the reported experimental values (Edeling et al, 2014a). All these studies imply that there is a noticeable uncertainty in these coefficients and as demonstrated in (Edeling et al, 2014b), best flow-independent values for these coefficients are unlikely to exist. As described in (Pope, 2001), the default values of the closure coefficients in the standard $k-\varepsilon$ model are obtained from a compromise so as to enable the model to perform for a variety of the airflow problems.

In Table 1, a number of studies associated with the effect of the closure coefficients for different physical problems are summarized. In an early work conducted by Duynkerke (1988), a set of modified closure coefficients for the standard $k-\varepsilon$ model was suggested based on a comparison between the RANS model and a measurement study and LES model over a flat terrain for neutral and stable atmospheric boundary layer conditions. He used Panofsky and Dutton (1984) data and calculated $C_{\mu}=0.033$, which is lower than its default value of 0.09 . He also proposed values of $C_{\varepsilon 1}=1.46$ and $C_{\varepsilon 2}=1.85$, which are close to their default values of 1.44 and 1.92, respectively. For Von Karman constant equal to 0.4 , he has also obtained $\sigma_{\varepsilon}=2.38$, which is greater than its default value of 1.3 used in most of the CFD solvers. For $\sigma_{k}$, the default value of 1 was assumed. In a similar work by Detering and Etling (1985), a modification on the $\varepsilon$ equation constants of the $k-\varepsilon$ model was adapted for mesoscale atmospheric boundary layer modeling above a flat and complex terrain. 
Table 1 The value of closure coefficients for different flow problems

\begin{tabular}{|c|c|c|}
\hline Ref & Physical model & Closure coefficients \\
\hline $\begin{array}{l}\text { Launder and } \\
\text { Spalding (1974) }\end{array}$ & Free turbulent flows & $\begin{array}{c}C_{\varepsilon 1}=1.44, C_{\varepsilon 2}=1.92, C_{\mu}=0.09 \\
\sigma_{\varepsilon}=1.3, \sigma_{k}=1\end{array}$ \\
\hline $\begin{array}{l}\text { Mohamed and } \\
\text { LaRue (1990) }\end{array}$ & Grid-generated turbulence & $C_{\varepsilon 2}=1.77$ \\
\hline $\begin{array}{c}\text { Kim et al } \\
(1987)\end{array}$ & Fully developed channel flow & $0.06 \leq C_{\mu} \leq 0.095$ \\
\hline Pope (2001) & $\begin{array}{l}\text { Fully developed channel flow in log- } \\
\text { law region }\end{array}$ & $\sigma_{\varepsilon}=\frac{\kappa^{2}}{C_{\mu}^{1 / 2}\left(C_{\varepsilon 2}-C_{\varepsilon 1}\right)}$ \\
\hline $\begin{array}{l}\text { Duynkerke } \\
\text { (1988) }\end{array}$ & Neutral and Stable ABL & $\begin{array}{c}C_{\varepsilon 1}=1.46, C_{\varepsilon 2}=1.83, C_{\mu}=0.033 \\
\sigma_{\varepsilon}=2.38, \sigma_{k}=1\end{array}$ \\
\hline $\begin{array}{l}\text { Detering and } \\
\text { Etling (1985) }\end{array}$ & Neutral and Stable ABL & $\begin{array}{c}C_{\varepsilon 1}=1.13, C_{\varepsilon 2}=1.9, \sigma_{\varepsilon}=1.29, \sigma_{k}= \\
0.74\end{array}$ \\
\hline $\begin{array}{l}\text { Glover et al } \\
\text { (2011) }\end{array}$ & Idealized street canyon & $\begin{array}{c}C_{\varepsilon 1}=1, C_{\varepsilon 2}=2.2, C_{\mu}=0.12, \sigma_{\varepsilon}= \\
0.42, \sigma_{k}=0.462\end{array}$ \\
\hline $\begin{array}{l}\text { Edeling et al } \\
(2014 \mathrm{~b})\end{array}$ & $\begin{array}{l}\text { Wall-bounded flow with different } \\
\text { favorite and adverse pressure gradient }\end{array}$ & Case dependent \\
\hline $\begin{array}{l}\text { Guillas et al } \\
\text { (2014) }\end{array}$ & Idealized street canyon & $\begin{array}{c}C_{\varepsilon 1}=1, C_{\varepsilon 2}=2.2, C_{\mu}=0.12, \sigma_{\varepsilon}= \\
0.42, \sigma_{k}=0.462\end{array}$ \\
\hline $\begin{array}{l}\text { Zahid Iqbal and } \\
\text { Chan (2016) }\end{array}$ & High-raised cross-shaped buildings & $\begin{array}{c}C_{\varepsilon 1}=1, C_{\varepsilon 2}=1.92, C_{\mu}=0.12 \\
\sigma_{\varepsilon}=0.5, \sigma_{k}=0.53\end{array}$ \\
\hline
\end{tabular}

125

Due to the inherent uncertainty in the value of the closure coefficients (Mohamed and LaRue, 1990; Tavoularis and Karnik, 1989), some studies, therefore, considered these coefficients as uncertain variables; investigated the sensitivity of the RANS model outputs to the variability of the closure coefficients. For example, Dunn et al (2011), Glover et al (2011), Todd and Robert (2011), Cheung et al (2011), Edeling et al (2014b) and Guillas et al (2014) investigated the uncertainty in relation to the closure coefficients of the $\varepsilon$ equation, and discussed the applicability of statistical analysis for improving the accuracy of RANS models. Dunn et al (2011) studied the uncertainty in relation to the $k-\varepsilon$ coefficients using the Latin Hypercube Sampling (LHS) method through considering different forms of probability density function (PDF) for the closure coefficients. They demonstrated that the highest uncertainty of the flow parameters occurs in the recirculating region and near the reattachment point. Furthermore, a Bayesian calibration approach was introduced in (Cheung et al, 2011) in which coefficients of Spalart-Allmaras model (Spalart and Allmaras, 1992) were calibrated for a set of incompressible CFD models over a flat plate. Experimental data for the velocity profile and wall shear stress were used in the calibration process. In a similar work, Edeling et al (2014b) performed 13 separate Bayesian calibrations using the experimental velocity profile for 13 different pressure gradients. They used a two-dimensional compressible boundary layer program instead of a full RANS code in order to reduce the runtime and avoid surrogate model. Their results showed a noticeable variation of coefficient posteriors for the considered range of the flow for $C_{\varepsilon 2}$ and $C_{\mu}$.

In another work performed by Guillas et al (2014), a Bayesian calibration of the $k-\varepsilon$ closure coefficients for a flow in a street canyon was presented. They calibrated a CFD RANS model against a series of wind tunnel experiments (Kastner-Klein et al, 2001), and considered the turbulent kinetic 
energy distribution between regular street canyons as the quantity of interest. Uniform priors for closure coefficients, including $C_{\mu}, C_{\varepsilon 1}, C_{\varepsilon 2}$, and $\sigma_{k}$, were considered in their method and it was concluded that the $C_{\mu}$ values higher than 0.12 have the highest probability to better match the experimental data. For $\sigma_{k}$, values close to 0.5 were reported to be favorable. Solazzo (2008) reported a similar trend and showed that the lower values for $\sigma_{k}$ and $\sigma_{\varepsilon}$ than their default values can result in a better distribution of $k$ inside the street canyon, and thus improve the accuracy of the $k-\varepsilon$ model for such applications. In a recently published work by Zahid Iqbal and Chan (2016), a numerical and experimental analysis for the pedestrian wind environment around a group of high-rise cross-shaped buildings was presented. They used the closure coefficients proposed by Guillas et al (2014) and performed two experimental test cases to modify these coefficients. Their modified values (see Table 1) showed a better agreement with the experimental results relative to the default values for the standard $k-\varepsilon$ model.

This article aims to propose a systematic way to find the optimized values for the closure coefficients of RANS family turbulence models to improve the accuracy of CFD simulations for microclimate and urban studies. The methodology is based on a stochastic optimization approach and the Monte Carlo Sampling (MCS) technique, which is later applied to a case study to demonstrate the capability of the developed approach. Although the stochastic optimization and MCS method have been widely used for the reliability-based design and robust optimization of complex systems (Shah et al, 2015; Tang and Périaux, 2012), their application for calibration of the closure coefficients for ABL flow modeling is a novel approach. The proposed method in this study requires fewer samples (CFD simulations) than the previous calibration methods based on the Bayesian approaches. The case study considered in this article is the airflow around a high-rise building in a non-isothermal ABL in which optimized closure coefficients for the $k-\varepsilon$ model were investigated. A constant value for the turbulent Prandtl number was taken into consideration during the optimization. The experimental data of the airflow behind a high-rise building in an unstable non-isothermal turbulent flow by Yoshie et al (2011) were used in the calibration process to define various validation metrics. Using the MCS technique and stochastic optimization, a set of new closure coefficients will be obtained and accordingly they can improve the accuracy of the turbulence model. Numerical data for velocity in the wake region behind the building will be considered as the objectives of the optimization technique.

\section{Methodology}

The main objective of this study is to propose a systematic way to improve the accuracy of the RANS models in microclimate studies; it is achieved through modifying the closure coefficients of turbulence models using a stochastic optimization approach. To this end, a parametric sensitivity analysis will be performed at the first step to investigate the impact of the model coefficients on the accuracy of the CFD model. In the next step, the model coefficients will be inserted into an optimization module as a set of uncertain variables, and eventually, the best range of the coefficients will be calculated so accurately that the highest agreement between the experiment and CFD results can be achieved. 


\subsection{Optimization procedure}

Stochastic optimization approaches can be used in models in which exact data are unknown, but bounded by a set of realization or scenarios (Goerigk and Schöbel, 2016). This is the case in RANS turbulence models where the numerical values of the closure coefficients are chosen through combination of heuristic and empirical decision making (Schaefer et al, 2016). Thus, RANS coefficients can be considered as epistemic uncertainty variables with a uniform probability density function (PDF) to provide an equal probability for all the values in the interval to be an optimum candidate (Guillas et al, 2014). The concept of stochastic optimization used in this study, known as a robust optimization method, is described in (Van der Velden and Koch, 2010).

The brief description of the formulation of stochastic optimization can be mathematically stated as finding a set of design variables $\mathrm{X}$ that (Koch et al, 2004):

$$
\begin{array}{ll}
\text { Minimize: } & f\left(\mu_{y}(X), \sigma_{y}(X)\right) \\
\text { Subject to: } & g_{i}\left(\mu_{y}(X), \sigma_{y}(X)\right) \leq 0 \\
& X_{L} \leq X \leq X_{U}
\end{array}
$$

where $X_{L}$ and $X_{U}$ are the lower and upper limits for input parameter $X$. In this formulation, the output constraint $g_{i}$ is expressed in terms of mean value and standard deviation. A weighted sum approach was used to define the objective function, which includes a term for mean value variation relative to the target and a term to minimize the response variation (Koch et al, 2004):

$$
F=\sum_{i=1}^{l}\left[\frac{w_{1_{i}}}{s_{1_{i}}}\left(\mu_{y_{i}}-M_{i}\right)^{2}+\frac{w_{2_{i}}}{s_{2_{i}}} \sigma_{y_{i}}^{2}\right]
$$

where $w_{1_{i}}$ and $w_{2_{i}}$ are the weighting factors, and $s_{1_{i}}$ and $s_{2_{i}}$ are the scale factors related to each term. The weighting factors determine the importance of each objective while the scaling factors are used to normalize the objectives. $M_{i}$ stands for the target of the output response $i$ and $l$ is the total number of output responses. The statistical variability of output responses (i.e. $\mu_{y_{i}}$ and $\sigma_{y_{i}}$ ), which are required by the stochastic optimization formulation, can be estimated using the Monte Carlo simulation (MCS) technique.

In Fig. 2, a schematic of the optimization process for calibrating the closure coefficients is shown. By coupling the Monte Carlo sampling technique and CFD model, input variables (closure coefficients) randomly vary in accordance with their given PDFs. CFD model will be repeatedly run to characterize the statistical parameters of the output values (i.e. validation metrics), including their mean and standard deviation values. By integrating the Monte Carlo sampling into an optimizer, not only can the best mean value of the desired outputs (validation metrics) be calculated, but it is also possible to minimize the standard deviation of the output values so as to reduce the effects of uncertainty of the input variables on the output response. Nonlinear Programing with Non-Monotone and Distributed Line Search (NLPQLP) optimization method (Schittkowski, 2006), a well suited method for highly non-linear design spaces, was used for the optimization purpose. A descriptive sampling technique (Tari and Dahmani, 2006) was used for MCS, which is more efficient than the conventional simple random sampling method (Koch et al, 2004). 50 samples were considered for the MCS during each optimization iteration. Different objective functions can be defined for the 
optimization process, including the means and standard deviation of validation metrics, which depend 222 on the availability of the experimental data for each specific case.

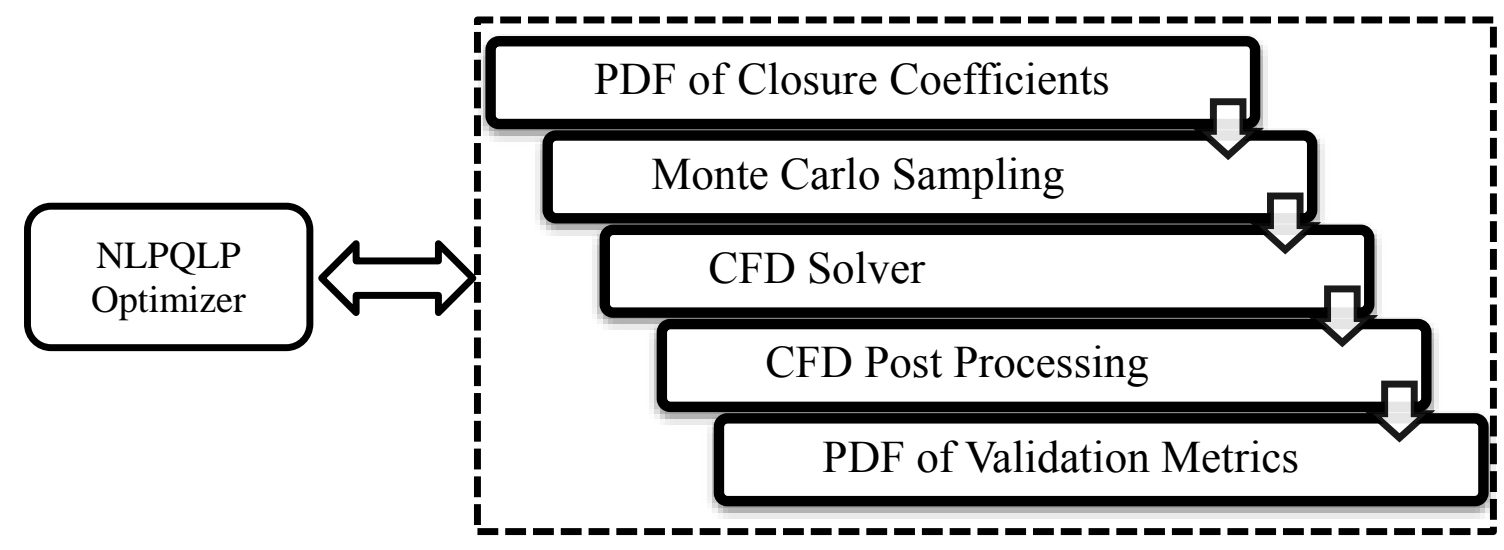

\subsection{Mathematical modeling}

The 3D steady Reynolds averaged Navier-Stokes (RANS) equations were used to simulate the airflow around the building. These equations can be derived by substituting mean and fluctuating components of the airflow variables into the Navier-Stokes equations (CFX, 2011):

$$
\begin{aligned}
& \frac{\partial\left(\rho U_{j}\right)}{\partial x_{j}}=0 \\
& \frac{\partial}{\partial x_{j}}\left(\rho U_{i} U_{j}\right)=-\frac{\partial P}{\partial x_{i}}+\frac{\partial}{\partial x_{j}}\left(\tau_{i j}-\rho \overline{u_{\imath} u_{J}}\right)+S_{M_{i}}
\end{aligned}
$$

where $U_{i}$ is the average velocity and $u_{i}$ is the fluctuating velocity. $\tau_{i j}$ is the viscous stress tensor (including both normal and shear components of the stress tensor) and $S_{M_{i}}$ is the sum of body forces. The Boussinesq model was used in this study. Temperature field was also calculated by solving the energy equation while eddy diffusivity was used to model turbulent energy fluxes (CFX, 2011):

$$
\frac{\partial}{\partial x_{j}}\left(\rho U_{j} h_{\text {total }}\right)=\frac{\partial}{\partial x_{j}}\left(\lambda \frac{\partial T}{\partial x_{j}}+\frac{\mu_{t}}{P r_{t}} \frac{\partial h}{\partial x_{j}}\right)+\frac{\partial}{\partial x_{j}}\left[U_{i}\left(\tau_{i j}-\rho \overline{u_{\imath} u_{\jmath}}\right)\right]+U_{j} S_{M_{j}}
$$

where $\lambda$ is the thermal conductivity of air and $P r_{t}$ is the turbulent Prandtl number, which has a constant value of 0.9. $U_{j} S_{M_{j}}$ represents the work due to the external momentum source. $h_{\text {total }}$ is the total enthalpy and is related to the static enthalpy $(h)$ by:

$h_{\text {total }}=h+\frac{1}{2} U^{2}$

Air was considered to be incompressible, which is reasonable for atmospheric boundary layer (ABL) flows (Richards and Norris, 2011); the air density, specific heat capacity at constant pressure, and thermal expansion coefficient were considered to be $1.185 \mathrm{~kg} / \mathrm{m}^{3}, 1004.4 \mathrm{j} / \mathrm{kg} \mathrm{K}$, and $0.0033561 / K$. The temperature was calculated from the static enthalpy as follows: 
$h-h_{\text {ref }}=C_{P}\left(T_{\text {static }}-T_{\text {ref }}\right)$

239 where $T_{r e f}=25^{\circ} \mathrm{C}$ is the reference temperature and $h_{r e f}$ is the reference enthalpy which is zero at the 240 reference temperature.

In this study the $k-\varepsilon$ turbulence model with the Kato-Launder modification (Kato and Launder, 1993) was used, which is based on the eddy viscosity hypothesis in which Reynolds stresses can be

244 hypothesis as follows:

$$
-\rho \overline{U_{l} U_{J}}=\mu_{t}\left(\frac{\partial U_{i}}{\partial x_{j}}+\frac{\partial U_{j}}{\partial x_{i}}\right)-\frac{2}{3} \delta_{i j} \rho k
$$

245 where $\mu_{t}$ is the eddy viscosity or turbulent viscosity, which can be defined as below:

$$
\mu_{t}=C_{\mu} \rho \frac{k^{2}}{\varepsilon}
$$

For the $k-\varepsilon$ model, values of $k$ and $\varepsilon$ come directly from their differential transport equations (Mori et al, 1995):

$$
\begin{aligned}
& \frac{\partial \rho U_{j} k}{\partial x_{j}}=\frac{\partial}{\partial x_{j}}\left[\left(\mu+\frac{\mu_{t}}{\sigma_{k}}\right) \frac{\partial k}{\partial x_{j}}\right]+P_{k}-\rho \varepsilon+P_{k b} \\
& \frac{\partial \rho U_{j} \varepsilon}{\partial x_{j}}=\frac{\partial}{\partial x_{j}}\left[\left(\mu+\frac{\mu_{t}}{\sigma_{\varepsilon}}\right) \frac{\partial \varepsilon}{\partial x_{j}}\right]+\frac{\varepsilon}{k}\left(C_{\varepsilon 1} P_{k}-C_{\varepsilon 2} \rho \varepsilon+C_{\varepsilon 1} P_{\varepsilon b}\right)
\end{aligned}
$$
where $P_{k}$ is the production of turbulence due to shear, which is modified by Kato and Launder (1993):

$P_{k}=\rho C_{\mu} \varepsilon S \Omega$

249 where $S$ and $\Omega$ are respectively the dimensionless strain and vorticity parameters, which are calculated 250 as below:

$$
\begin{aligned}
& S=\frac{k}{\varepsilon} \sqrt{\frac{1}{2}\left(\frac{\partial U_{i}}{\partial x_{j}}+\frac{\partial U_{j}}{\partial x_{i}}\right)^{2}} \\
& \Omega=\frac{k}{\varepsilon} \sqrt{\frac{1}{2}\left(\frac{\partial U_{i}}{\partial x_{j}}-\frac{\partial U_{j}}{\partial x_{i}}\right)^{2}}
\end{aligned}
$$

$251 P_{k b}$ and $P_{\varepsilon b}$ are buoyancy turbulence production and dissipation terms, respectively:

$$
\begin{aligned}
P_{k b} & =\frac{\mu_{t}}{\sigma_{p}} \beta g_{i} \frac{\partial T}{\partial x_{i}} \\
P_{\varepsilon b} & =\max \left(0, P_{k b}\right)
\end{aligned}
$$

252 where $\sigma_{p}=0.9$ is the turbulent Schmidt Number and $\beta$ is the thermal expansion coefficient. Values of 253 the closure coefficients, according to (Launder and Spalding, 1974), are predefined as the default 254 values for most of the popular CFD tools as below:

$$
C_{\mu}=0.09, C_{\varepsilon 1}=1.44, C_{\varepsilon 2}=1.92, \sigma_{k}=1, \sigma_{\varepsilon}=1.3
$$




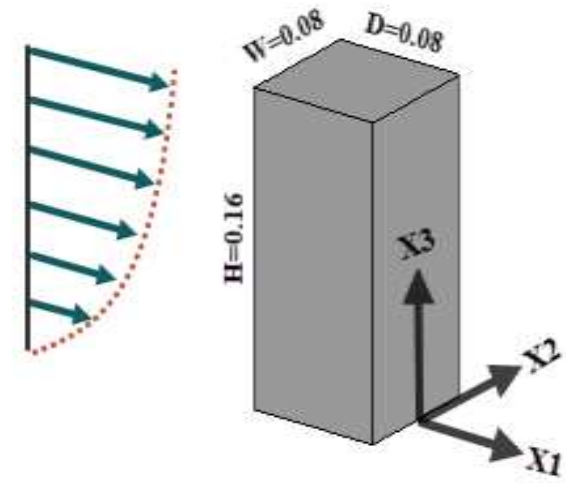

(a)

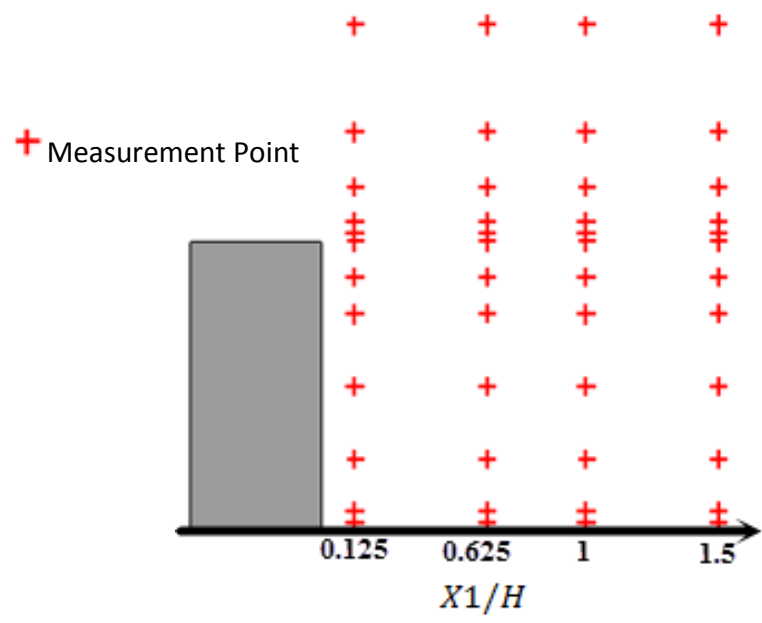

(b)

Figure 3 Schematic of Yoshie et al (2011) experiment: (a) bluff body dimensions, (b) measurement points

\section{CFD Simulation}

The RANS equations were solved using the commercial software ANSYS CFX, which uses an element-based finite volume discretization method.

\subsection{Description of the wind tunnel experiment for unstable ABL}

As seen in Fig.3, the experimental data for the closure coefficients optimization were taken from Yoshie et al (2011) in which a detailed experimental analysis on airflow and gas dispersion was conducted around a high-rise building in a non-isothermal ABL. The target building had a dimension of $W \times D \times H=0.08(\mathrm{~m}) \times 0.08(\mathrm{~m}) \times 0.16(\mathrm{~m})$, which was placed in an atmospheric wind tunnel at Tokyo Polytechnic University. The surface of the wind tunnel had a uniform temperature of $45.3^{\circ} \mathrm{C}$ while the air velocity and temperature at the inlet were reported $U_{H}=1.37 \frac{\mathrm{m}}{\mathrm{s}}$ and $\theta_{H}=11^{\circ} \mathrm{C}$, respectively.

\subsection{Computational domain, grid, and boundary conditions}

A rectangular computational domain, as shown in Fig.4, was considered for the isolated building case based on the recommendations by AIJ guidelines (Tominaga et al, 2008) and similar studies (Mirzaei and Carmeliet, 2013). The domain width, length, and height were $1.2(\mathrm{~m}) \times 2(\mathrm{~m}) \times 1(\mathrm{~m})$. ICEM CFD meshing package was used to create structured hexahedral mesh applying the blocking technique. A grid-sensitivity analysis was conducted for three different mesh numbers with 229,401; 396,864; and 686,585 cells as coarse, medium and fine mesh configurations. Results showed a very negligible difference, less than $1 \%$, between the prediction of the velocity profile in the wake region for the medium and fine meshes; hence the medium mesh configuration was selected for the study. Number of the cells around the building block was $30 \times 30 \times 45$. An O-grid block with first-layer size of $1.3 \times 10^{-4}(\mathrm{~m})$ was used around the building, which resulted to an average $y^{+} \approx 1$ for the solid surfaces. No-slip boundary condition was considered for all solid walls and a constant temperature boundary condition was applied to the ground surface. All solid walls were treated as smooth walls. Symmetric wall boundary condition was considered for the lateral boundaries while a free-slip wall boundary condition was assumed for the top boundary surface. Zero static pressure was applied at the outlet plane. Inlet boundary condition for the vertical velocity, temperature and turbulent 
kinetic energy profiles were also obtained directly from Yoshie et al (2011) experiment. Turbulent 284 kinetic energy dissipation rate $\varepsilon(z)$ was also approximated from the below equation (Yoshie et al, 285 2011):

$\varepsilon(z)=\overline{u_{1} u_{3}} \frac{\partial U_{1}}{\partial x_{3}}-g_{3} \beta \overline{u_{3} \theta^{\prime}}$

where $\overline{u_{3} \theta^{\prime}}$ is the turbulent heat flux obtained from the experiment. Vertical distribution of the time averaged streamwise velocity, turbulent kinetic energy, and temperature are depicted in Fig. 5.
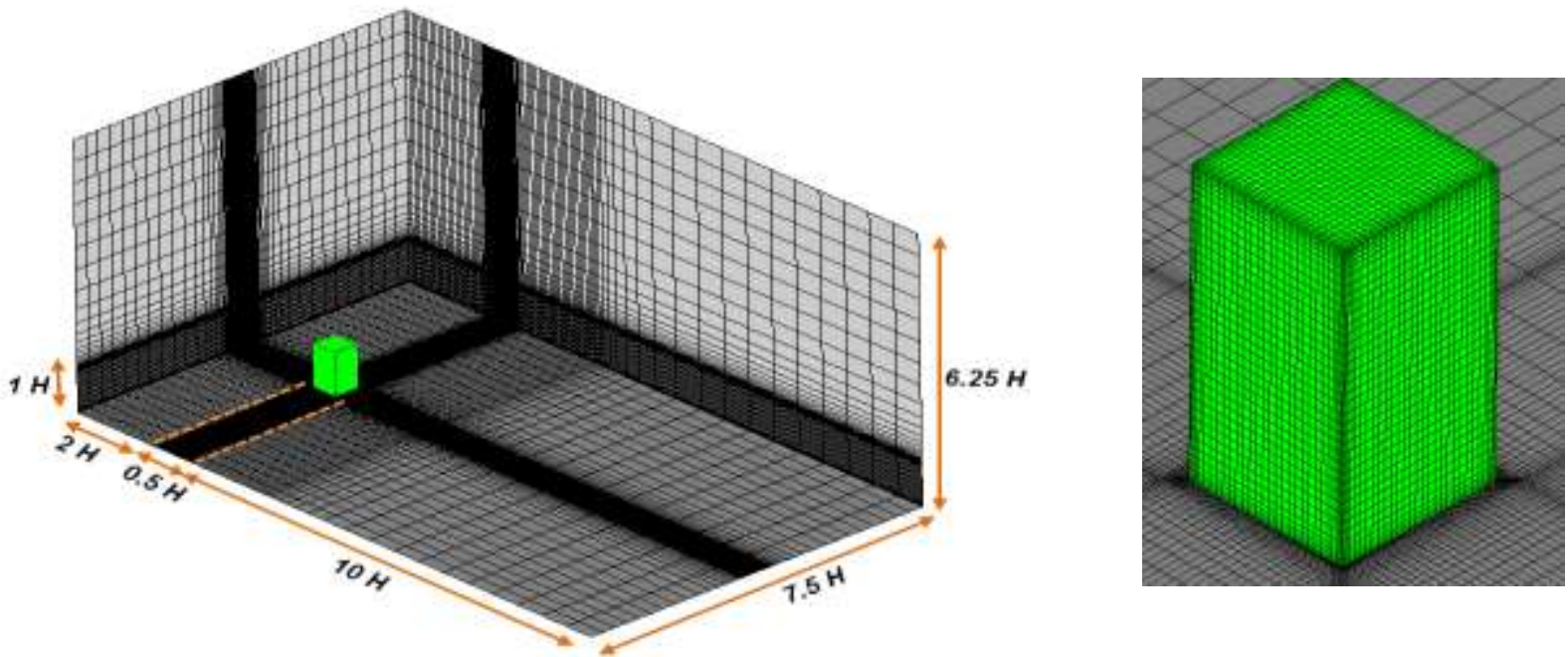

Figure 4 Computational domain and grid arrangement

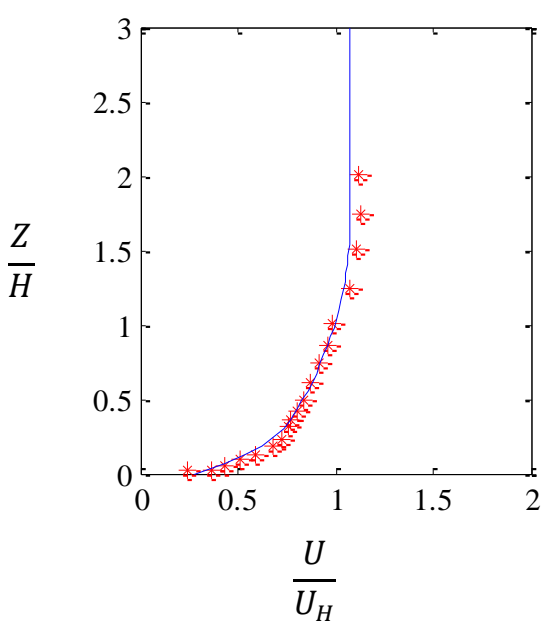

(a)

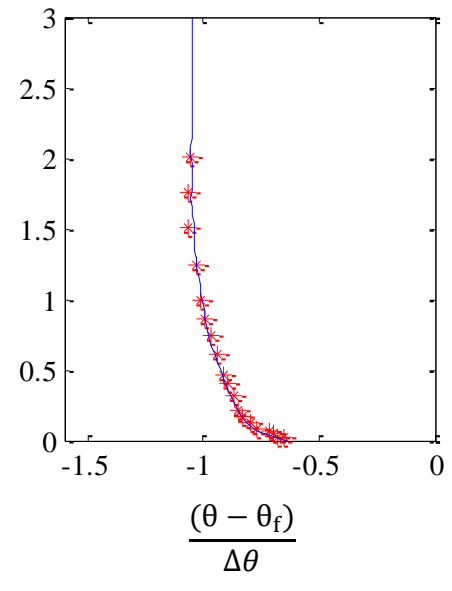

(b)

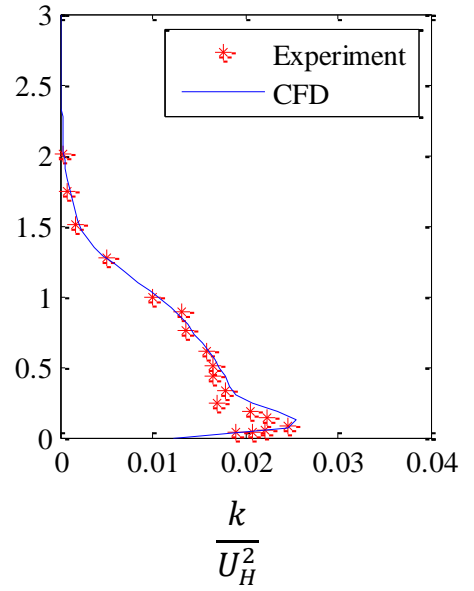

(c)

Figure 5 Inflow boundary condition for (Yoshie et al, 2011): (a) velocity, (b) temperature, (c) turbulent kinetic energy

\subsection{Solver setting}

Pressure-velocity coupling was based on the Rhie-Chow interpolation proposed by Rhie and Chow (1983) while a co-located grid layout was further used. The High Resolution Scheme was used for the discretization of the advection terms while tri-linear shape functions were used to evaluate the 
spatial derivatives for all the diffusion terms. For the near-wall treatment, scalable wall function based on the modification of the Launder and Spalding (1974) was used. The CFD solver iterations have been continued until reaching RMS residual of less than $10^{-5}$ for continuity, velocity components, energy, $k$ and $\varepsilon$ equations.

\section{Results}

In this section, results of the proposed systematic approach for a test case of the defined nonisothermal ABL flow around a building based on the $k-\varepsilon$ model will be presented. At first, results of a sensitivity analysis on the CFD model's response to the closure coefficients variation are presented. After that, the main outcomes of the optimization methodology are discussed.

\subsection{Sensitivity analysis of the CFD model response to the variation of the closure coefficients}

In order to find the effect of the closure coefficients variation on the response of the CFD model, a parametric sensitivity analysis has been initially conducted. Results of the parametric sensitivity analysis were then used to identify the influential parameters for being later used in the statistical optimization. As shown in (Dunn et al, 2011; Guillas et al, 2014), the highest uncertainty of flow parameters occurred in the recirculating region and near the reattachment point after the leeward side within the street canyon. Hence, in the case study, velocity data at 48 points in the wake region along four streamwise positions, i.e. $\frac{X 1}{H}=0.125, \frac{X 1}{H}=0.625, \frac{X 1}{H}=1$, and $\frac{X 1}{H}=1.5$, were selected as the target points for calculation of the validation metrics (see Fig.3 (b)).

Two validation metrics were adapted in this study to quantify the agreement between the experimental and numerical results. These metrics are namely the hit rate $q$ and the fraction of the predictions within a factor of two of the observations (FAC2) defined as follows (Tominaga, 2015):

$$
\begin{array}{ll}
q=\frac{1}{N} \sum_{i=1}^{N} n_{i} & \text { if }\left|\frac{P_{i}-Q_{i}}{P_{i}}\right| \leq D_{q} \text { or }\left|P_{i}-Q_{i}\right| \leq W_{q} \quad n_{i}=1 \quad \text { else } n_{i}=0 \\
F A C 2=\frac{1}{N} \sum_{i=1}^{N} n_{i} & \text { if } 0.5 \leq \frac{P_{i}}{Q_{i}} \leq 2 \quad n_{i}=1 \quad \text { else } \quad n_{i}=0
\end{array}
$$

where $Q_{i}$ and $P_{i}$ are the observed (measured) and predicted (computed) values of a given variable, respectively, and $N$ is the number of data points. The thresholds for $q$ are recommended $D_{q}=0.25$ and $W_{q}=0.03$ for streamwise velocity (Gousseau et al, 2013; Tominaga, 2015). For a complete agreement between the experimental and numerical results, the value of $q$ and $F A C 2$ should be 1 . To perform the parametric sensitivity study, four coefficients of the $k-\varepsilon$ turbulence model, i.e. $C_{\varepsilon 1}, C_{\varepsilon 2}$, $C_{\mu}$, and $\sigma_{k}$, were linearly altered while for each variable, a number of 20 uniformly distributed samples were selected among its interval. The value of $\sigma_{\varepsilon}$ was calculated using the eq. (21) for each set of the closure coefficients. In regard to the previous studies in literature, a range of closure coefficients was considered as depicted in Table 2.

$$
\sigma_{\varepsilon}=\frac{\kappa^{2}}{C_{\mu}^{1 / 2}\left(C_{\varepsilon 2}-C_{\varepsilon 1}\right)}
$$




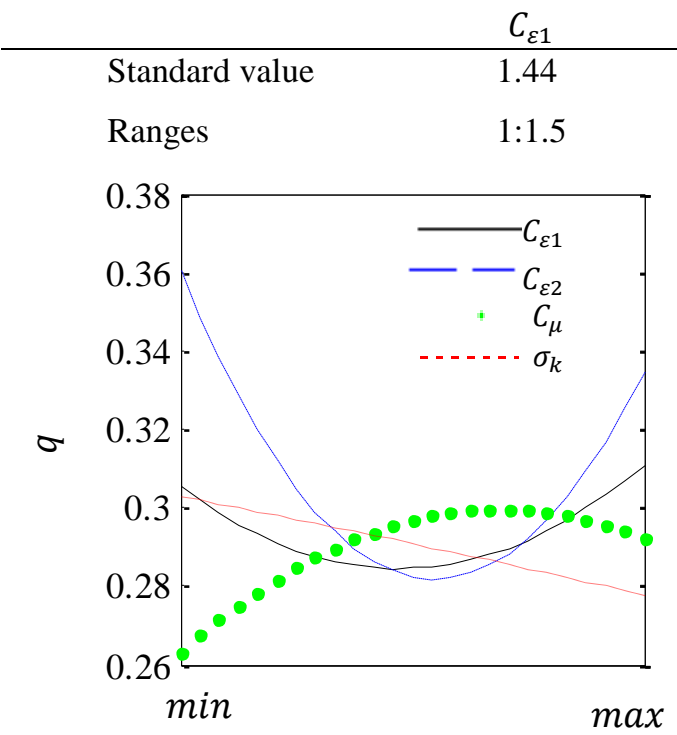

(a)

$\begin{array}{ccc}C_{\varepsilon 2} & \sigma_{k} & C_{\mu} \\ 1.92 & 1 & 0.09\end{array}$

$1.5: 3$

$0.8: 1.4$

0.05:0.15

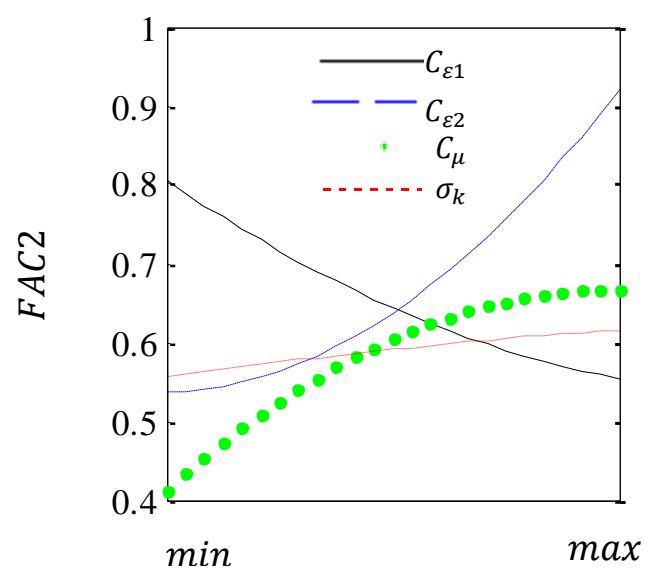

(b)

Figure 6 Variation of the validation metrics for the streamwise velocity component for isolated building case study: (a) hit rate $\boldsymbol{q}$, (b) $\mathbf{F A C 2}$

In Fig. 6, the variation of the validation metrics for the streamwise velocity against the closure coefficients is plotted. It can be seen that $C_{\varepsilon 2}$ and $C_{\mu}$ have a noticeable impact on both validation metrics, but $\sigma_{k}$ shows a lower impact. Considering the variation of FAC2, it reveals that the lower values of $C_{\varepsilon 1}$, namely values around 1 , provide a higher accurate results in terms of streamwise velocity distribution. In contrast, the higher values of $C_{\varepsilon 2}$, namely $C_{\varepsilon 2} \approx 3$, show a better agreement with the experiment. Same trend can be seen for $C_{\mu}$ where higher value for $F A C 2$ is obtained for the higher values of $C_{\mu}$, ranging between 0.11 and 0.15 . Both validation metrics seem to be less sensitive to $\sigma_{k}$ for this data set. Nonlinear variation of $q$ and FAC2 shows the necessity of using an optimization technique to systematically find optimal coefficients. It can be concluded that the default values for the closure coefficients, as shown in (Edeling et al, 2014b; Guillas et al, 2014), are not accurate for the considered test case with a strong wake region.

To demonstrate the effect of the closure coefficients on the turbulent kinetic energy distribution at the wake region behind the building, contours of $k / U_{H}^{2}$ are depicted in Fig. 7 obtained from the default value and three other cases of the closure coefficient values in addition to the experimental results by Yoshie et al (2011). It can be seen that for the reference case, which corresponds to the case with default value for the closure coefficients, the level of the turbulent kinetic energy inside the wake region behind the building is considerably low. For default closure coefficients, not only is the large mixing process behind the building underestimated, but the generation of $k$ over the roof is also underpredicted. For the case with $C_{\varepsilon 1}=1$, distribution level of $k$ inside the wake region is noticeably increased. Same improvement in the distribution of $k$ inside the wake region is observed for the case specified with $C_{\varepsilon 2}=3$. A minor improvement can be also seen for the case with $C_{\mu}=0.15$. For the cases with $C_{\varepsilon 1}=1$ and $C_{\varepsilon 2}=3$, the position of the formation of the high turbulent kinetic energy over the roof has changed in a way that is much closer to the experiment in comparison to the case with default coefficient values. Over the roof area, the average of $k$ is noticeably increased and a more 
agreement with the experiment is found. Improving the prediction accuracy of the $k$ distribution both inside the wake region behind the building and the separation region over the roof leads to a better estimation of the reattachment lengths in these regions.

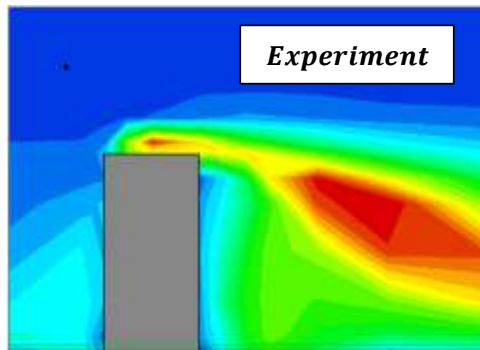

(a)

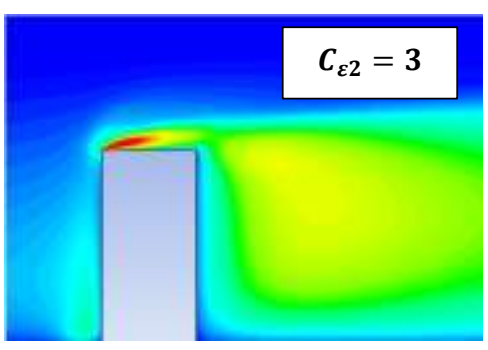

(d)

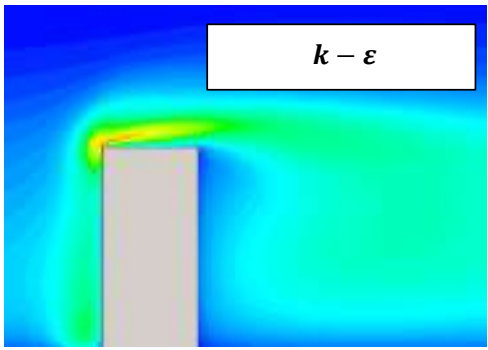

(b)

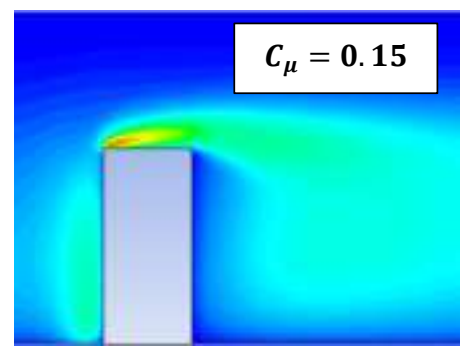

(e)

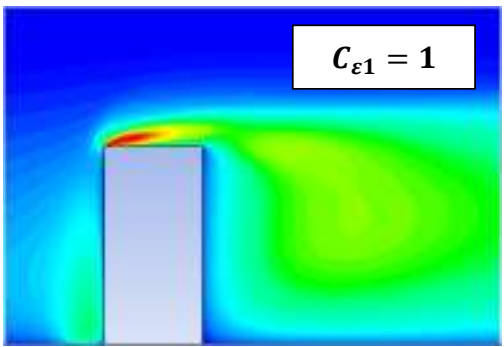

(c)

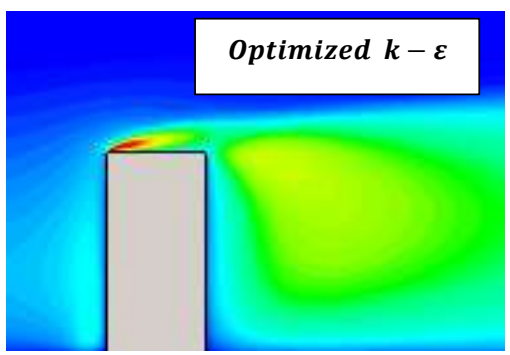

(f)

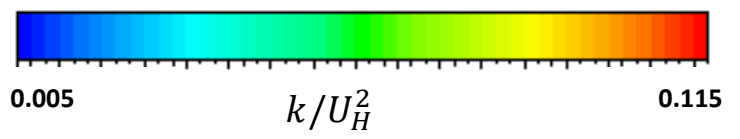
Figure 7 Contours of the turbulent kinetic energy: (a) experiments by Yoshie et al (2011), (b) $\boldsymbol{k}-\boldsymbol{\varepsilon}$, (c)
\[ C_{\varepsilon 1}=\mathbf{1} \text {, (d) } C_{\varepsilon 2}=3 \text {, (e) } C_{\mu}=\mathbf{0 . 1 5} \text {, (f) optimized coefficients } \]

Predicted values for the roof reattachment length $\left(X_{r}\right)$ and the floor reattachment length $\left(X_{f}\right)$ are presented in Table 3. The experimental value for the reattachment length at the floor is estimated to be $X_{f}=0.096(\mathrm{~m})$. This value for the reference case with the default coefficients is $X_{f}=0.260(\mathrm{~m})$ while it is $X_{f}=0.138(\mathrm{~m})$ when $C_{\varepsilon 2}=3$. In the case of $C_{\mu}=0.15$, the $k$ distribution increased in relation to the reference case, but its increase is lower than that of altered $C_{\varepsilon 1}$ and $C_{\varepsilon 2}$. This resulted in a longer reattachment length of $X_{f}=0.201(\mathrm{~m})$. The shortest roof reattachment length is predicted for $C_{\varepsilon 1}=1$ followed by the case for $C_{\varepsilon 2}=3$. This value is not reported in the experiment, but it can be estimated to be around $X_{r} \approx 0.045(\mathrm{~m})$.

Table 3 Comparison of the reattachment length on roof $\left(\boldsymbol{X}_{r}\right)$ and reattachment length behind the building $\left(\boldsymbol{X}_{f}\right)$

\begin{tabular}{cccccccc} 
& Experiment & $\boldsymbol{k}-\boldsymbol{\varepsilon}$ & $\boldsymbol{C}_{\boldsymbol{\varepsilon} \mathbf{1}}=\mathbf{1}$ & $\boldsymbol{C}_{\boldsymbol{\varepsilon} \mathbf{2}}=\mathbf{3}$ & $\boldsymbol{C}_{\boldsymbol{\mu}}=\mathbf{0 . 1 5}$ & $\boldsymbol{\sigma}_{\boldsymbol{k}}=\mathbf{1 . 4}$ & $\begin{array}{c}\text { Optimized } \\
\boldsymbol{k}-\boldsymbol{\varepsilon}\end{array}$ \\
\hline $\boldsymbol{X}_{\boldsymbol{r}}$ & $\mathrm{NA}$ & 0.061 & 0.023 & 0.024 & 0.029 & 0.043 & 0.016 \\
$\boldsymbol{X}_{\boldsymbol{f}}$ & 0.096 & 0.260 & 0.159 & 0.138 & 0.201 & 0.228 & 0.123
\end{tabular}

In Fig. 8, contours of the temperature distribution around the building for different closure coefficients, which proved to have a positive effect on the validation metrics, are displayed and compared with the experimental data. It is important to note that a fixed turbulent Prandtl number was considered for all simulations. In the case of default closure coefficients, due to the poor mixing 
behind the building, the temperature diffusion inside the wake region is noticeably lower than that of the experimental observation. For the cases with $C_{\varepsilon 1}=1$ and $C_{\varepsilon 2}=3$, thanks to the higher diffusion of the momentum inside the wake region, the temperature distribution becomes more realistic and a very close agreement with the experimental data can be obtained. For the case with $C_{\mu}=0.15$ temperature distribution has insignificant improvement due to the lower diffusion of the momentum inside the wake region. Results of the parametric sensitivity study show that among the considered closure coefficients for the considered flow condition, all the coefficients except $\sigma_{k}$ have a significant impact on the accuracy of the $k-\varepsilon$ model in terms of velocity, turbulent kinetic energy, and

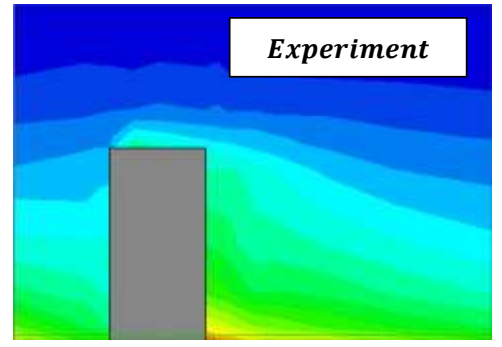

(a)

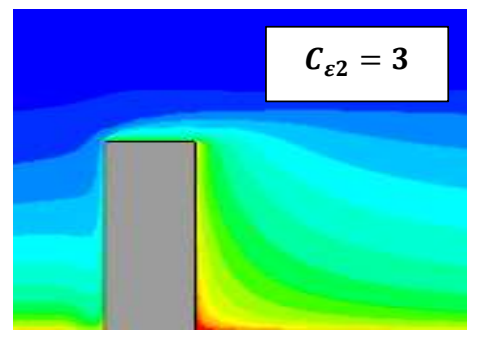

(d)

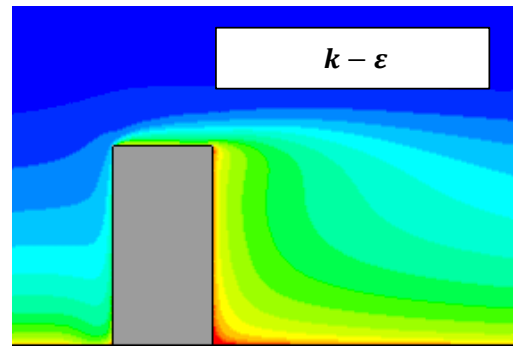

(b)

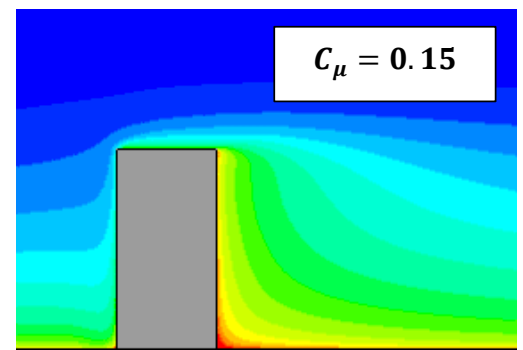

(e)
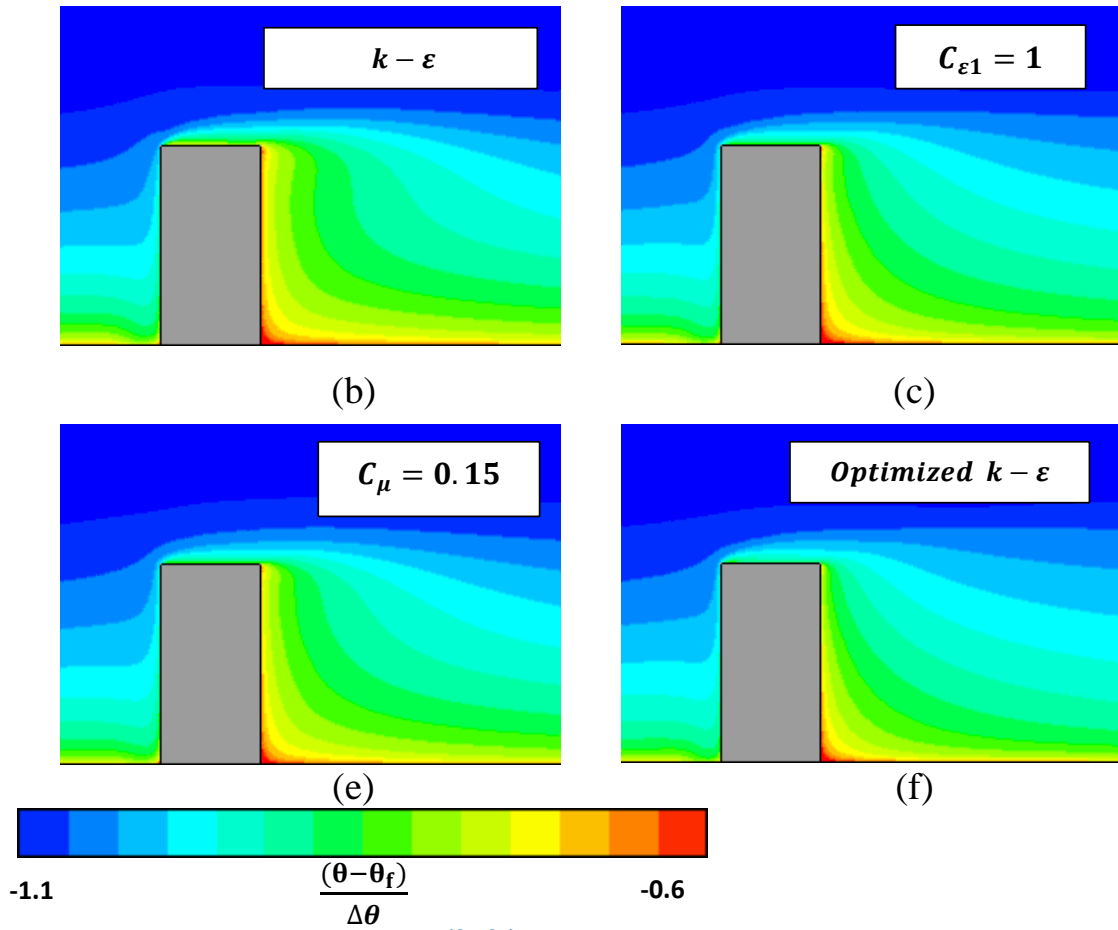

(c)

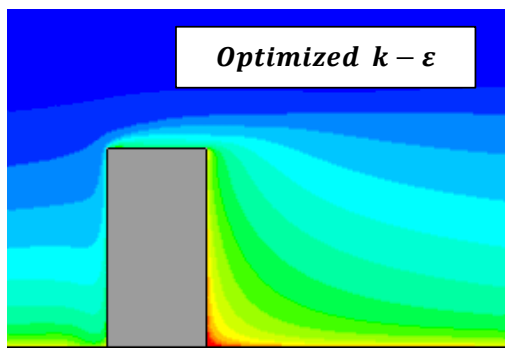

(f)

\subsection{Optimization results}

Based on the results of the parametric study, a stochastic optimization using the Monte Carlo sampling technique was performed to find out a modified set of closure coefficients, providing CFD results with a higher agreement with the experimental data in terms of the validation metrics defined in $\underline{\text { eq. (19) }}$ and eq. (20). In the stochastic optimization process, all input variables, including $C_{\varepsilon 1}, C_{\varepsilon 2}$ and $C_{\mu}$, were treated as the random or uncertainty variables with a uniform PDF ranged in accordance with the values in Table 2. $\sigma_{k}$ was not considered in the optimization as it has a low impact on the validation metrics according to the sensitivity parametric study (ig. 6, Fig. 7 and $\underline{\text { Fig. }}$ ) and it was set to its default value of 1 . Probability density function of $\sigma_{\varepsilon}$ was obtained during the optimization iterations based on eq. (21). The maximum iteration for the optimization loop was set to 100 while a termination accuracy of $10^{-6}$ was considered for optimization convergence. The objective functions 
of the both validation metrics, i.e. FAC2 and $q$, were considered to be maximized to reach an ideal value of 1 , which can be interpreted as the best agreement between the CFD simulations and experiment. An equal importance was considered for the mean and the standard deviation values of the validation metrics (FAC2 and $q$ ), hence a weighing factor of 1 was considered for $w_{1_{i}}$ and $w_{2_{i}}$ in eq. (2). The maximum value for FAC2 and $q$ is 1 and thus the values of the scaling factors $s_{1_{i}}$ and $s_{2_{i}}$ were set to 1 for all objectives in eq. (2).

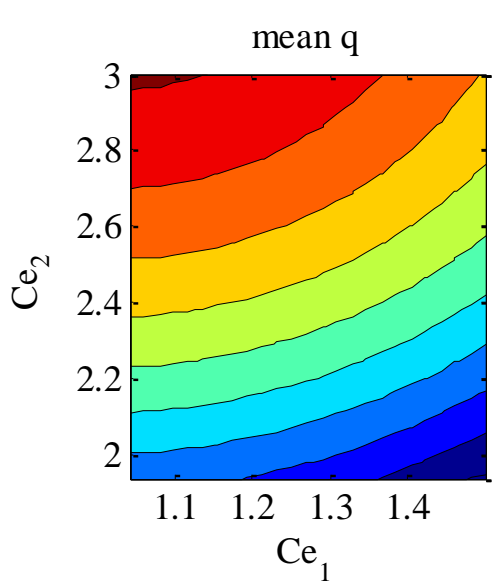

(a)

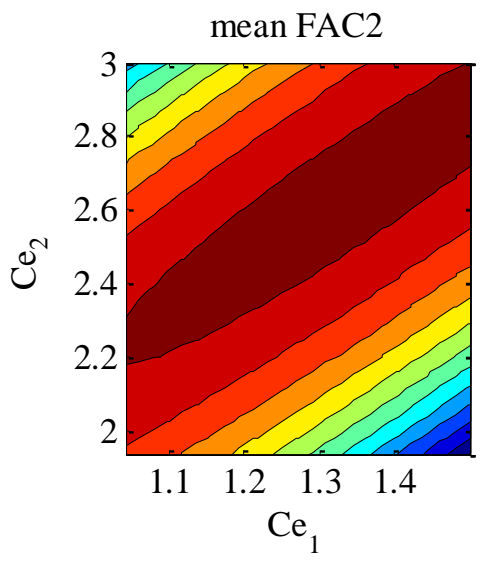

(d)

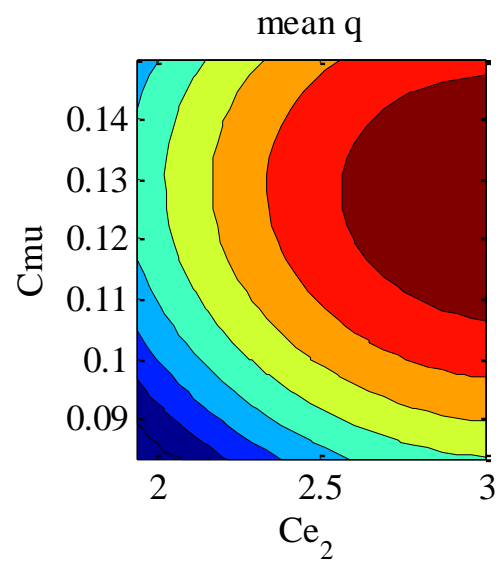

(b)

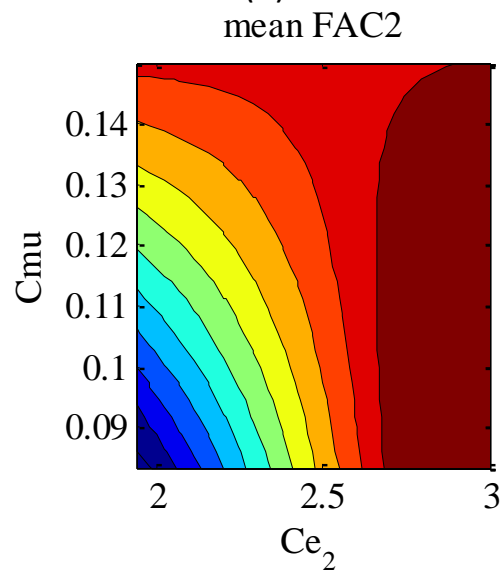

(e)

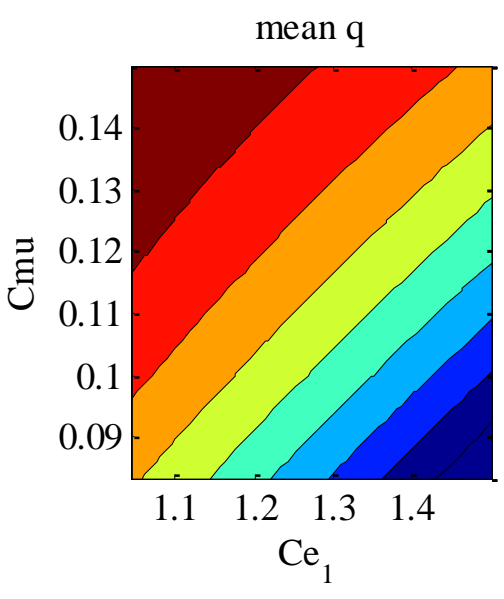

(c)

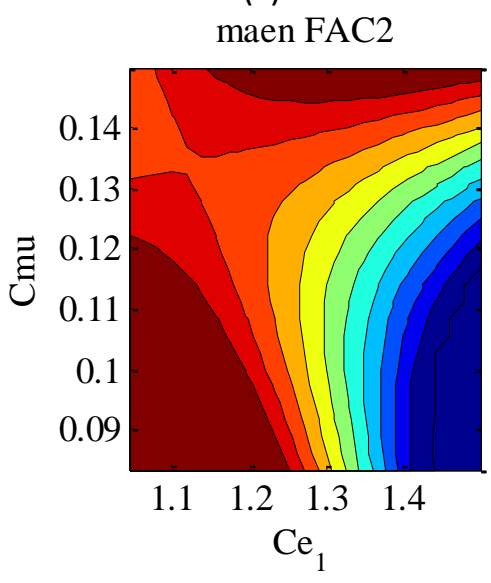

(d)

\section{Min}

Figure 9 Variation of the mean value of the validation metrics for the streamwise velocity component and the closure coefficients during the optimization process

Four objective functions were considered for the optimization process, including the mean values of $q$ and FAC2 and their standard deviations for the streamwise velocity component at 48 measurement points shown in Fig. 3 (b). The optimization process have been executed for $250 \mathrm{CFD}$ simulations. Variation of the mean value of the validation metrics for the streamwise velocity component and the closure coefficients during the optimization process is shown in Fig.9. Contours of hit rate $q$ show that the highest agreement between the CFD and the experiment occurs for $C_{\varepsilon 1}$ values in the range of $1.1 \leq C_{\varepsilon 1} \leq 1.3$ and for $C_{\varepsilon 2}$ values in the range of $2.6 \leq C_{\varepsilon 2} \leq 3$. The most suitable 
second validation metric ( $F A C 2$ ), a quite similar result is obtained. For $1.1 \leq C_{\varepsilon 1} \leq 1.5$ and $2.7 \leq$ $C_{\varepsilon 2} \leq 3, F A C 2$ has the highest value. The mean value of $F A C 2$ is acceptable for $C_{\mu}$ ranges between 0.12 and 0.15 . In general, it can be concluded that the highest probability of having a very close agreement between CFD results of the $k-\varepsilon$ model with those of the experimental analysis of nonisothermal airflow around a high-rise building in terms of the mean values of $q$ and FAC2 occurs for the closure coefficients in the ranges of $1.1 \leq C_{\varepsilon 1} \leq 1.5,2.7 \leq C_{\varepsilon 2} \leq 3$, and $0.12 \leq C_{\mu} \leq 0.15$. It is noteworthy to mention that the value of $\sigma_{k}$ is assumed as its default value of 1 while the value of $\sigma_{\varepsilon}$ can be calculated using eq. (21), which results in $0.32 \leq \sigma_{\varepsilon} \leq 0.56$.

As described earlier, not only were the mean values of the validation metrics considered in the stochastic optimization process, but their standard deviations were also included in the objective function to reduce the impact of the uncertainty of the closure coefficients on the validation metrics. Fig. 10 shows contours of the standard deviation of FAC2 for the streamwise velocity. It can be seen that in the specified ranges, where the mean values of the validation metrics are optimum, their standard deviations are also in their minimum values. Finally, the optimum values of the closure coefficients, resulted in the highest mean value for the validation metrics with the lowest standard deviation, can be expressed as follows:

$$
C_{\varepsilon 1}=1.489, C_{\varepsilon 2}=2.801, C_{\mu}=0.146, \sigma_{\varepsilon}=0.373, \sigma_{k}=1
$$
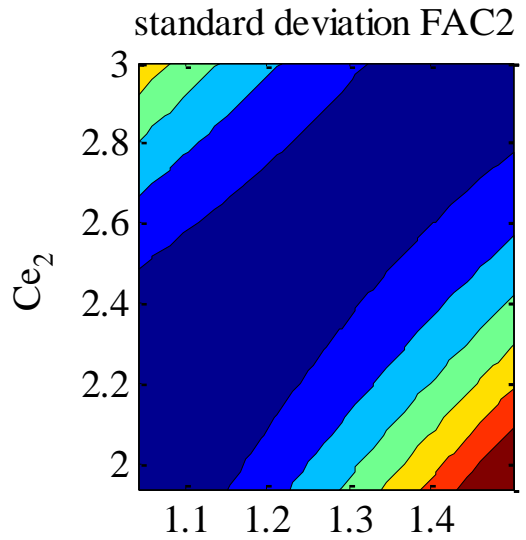

$\mathrm{Ce}_{1}$ standard deviation FAC2

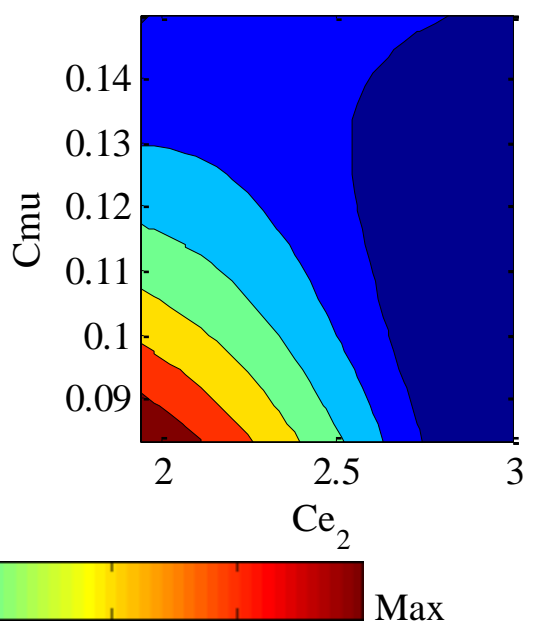

Figure 10 Plot of the standard deviation of the FAC2 for streamwise velocity in the optimization process

The mean values of validation metrics $q$ and FAC2 for streamwise velocity increased from 0.31 and 0.54 to 0.47 and 0.91 for default coefficients and optimized coefficients, respectively. The standard deviation of $q$ and FAC2 were also found to be 0.05 and 0.03 for the optimized coefficients. In general, using the modified closure coefficients in the $k-\varepsilon$ formulation results in a higher momentum mixing and turbulent kinetic energy inside the wake region behind the building. This was momentum diffusion is related to the value of $C_{\mu}$, rising from 0.09 to 0.14 , and increase of the TKE level inside the wake region. For the case considered in this study, when the modified closure 
coefficients were used, the average values of the momentum diffusion, $k$ diffusion, $k$ production term, $\varepsilon$ production term, and $\varepsilon$ dissipation term over the measurement points in the wake region grew about $40 \%, 51 \%, 52 \%, 32 \%$ and $34 \%$, respectively.

In order to observe the effect of the optimized closure coefficients on the airflow distribution around the building, results of the CFD simulation with the optimized closure coefficients are presented and discussed. In Fig. 11 (a) and Fig. 11(b), vertical distribution of the streamwise velocity $\frac{U}{U_{H}}$ at two locations behind the building, i.e. $\frac{X_{1}}{H}=0.625$ and $\frac{X_{1}}{H}=1$, are depicted for the reference CFD model with default closure coefficients as well as the optimized CFD model with the new set of the closure coefficients. The results are also compared to those reported in Yoshie et al (2011). For the reference case with the default coefficients, the reverse flow in the wake region is overestimated due to the poor momentum mixing behind the building. For the case with optimized closure coefficients, a significant improvement in the prediction accuracy of the velocity distribution in the wake region can be clearly observed, which results from a better momentum mixing. The reattachment length predicted for the default closure coefficients, as reported in Table 3, is $X_{f}=0.260(\mathrm{~m})$, which is much longer than that of the experiment with the value of $X_{f}=0.096(m)$. The predicted reattachment length behind the building for the optimized coefficients is $X_{f}=0.123(\mathrm{~m})$, appearing closer to the value of the measurement.

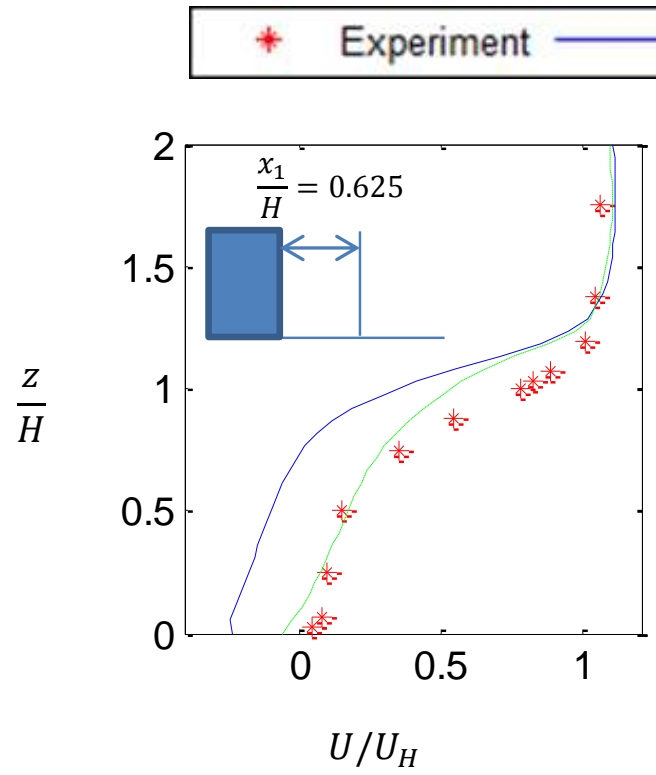

(a)

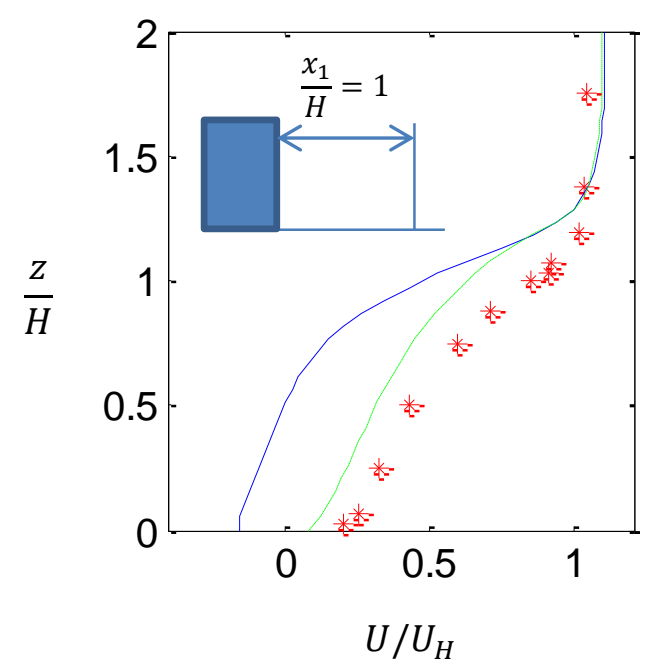

(b)

Figure 11 Vertical distribution of the streamwise velocity $\frac{\mathrm{U}}{\mathrm{U}_{\mathrm{H}}}$ in the wake region behind the building at:

$$
\text { (a) } \frac{X_{1}}{H}=0.625 \text {, (b) } \frac{X_{1}}{H}=1
$$

In the case of modified coefficients, distribution of turbulent kinetic energy along with the diffusion of TKE and its production term inside the wake region behind the building have been increased noticeably in comparison with the results obtained by the default coefficients (see Fig. 7). However, comparison between the experimentally measured turbulent kinetic energy (Fig. 7(a)) and those predicted by modified RANS model (Fig. 7(f)), shows that the CFD model significantly underpredicts the $k$ distribution behind the building. It refers to the fact that the steady RANS models are 
inherently incapable of calculating the unsteady nature of the turbulent kinetic energy because of the 464 large-scale fluctuations behind the building.

465

466

467

468

469

470

471

In Fig. 12, the horizontal distribution of the streamlines is depicted for the $k-\varepsilon$ model using default and optimized closure values. These streamlines are further compared with the results of the experimental and LES models presented in Yoshie et al (2011). A long recirculation region can be seen for the $k-\varepsilon$ model with the default coefficients. However, for the case with modified closure coefficients, the length of the recirculating region considerably decreases. The results hence show more agreement with the experimental data and LES; namely a more accurate, but computationally expensive model.

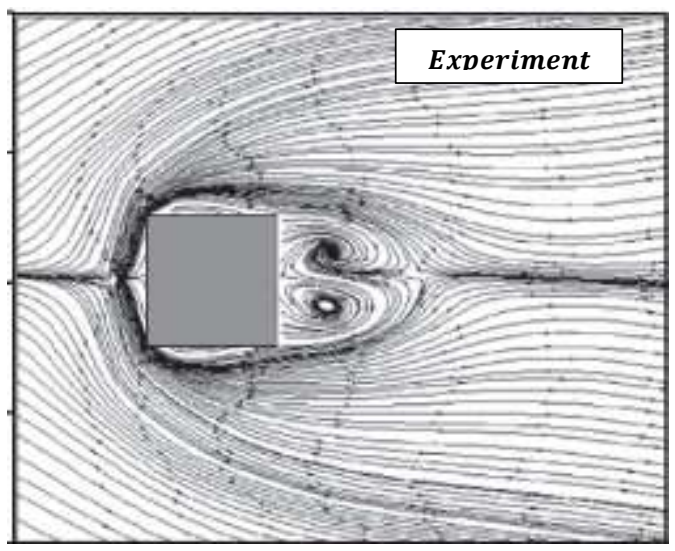

(a)

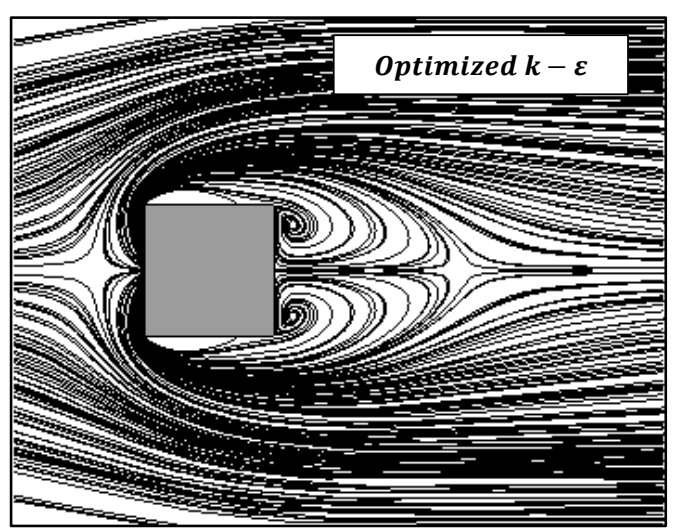

(c)

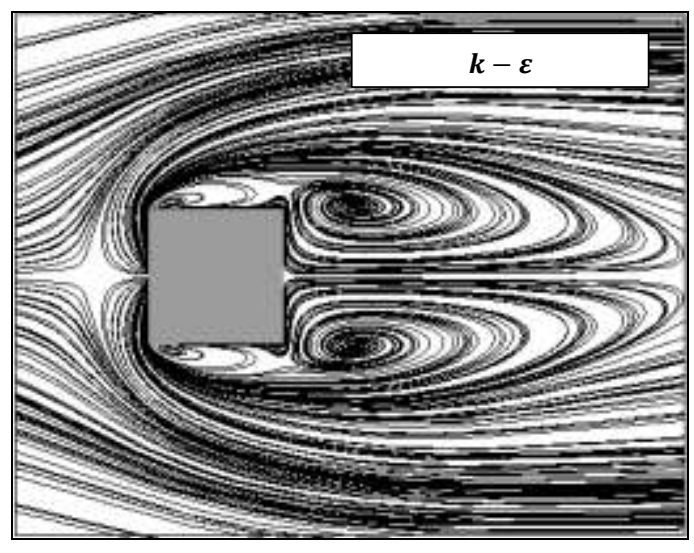

(b)

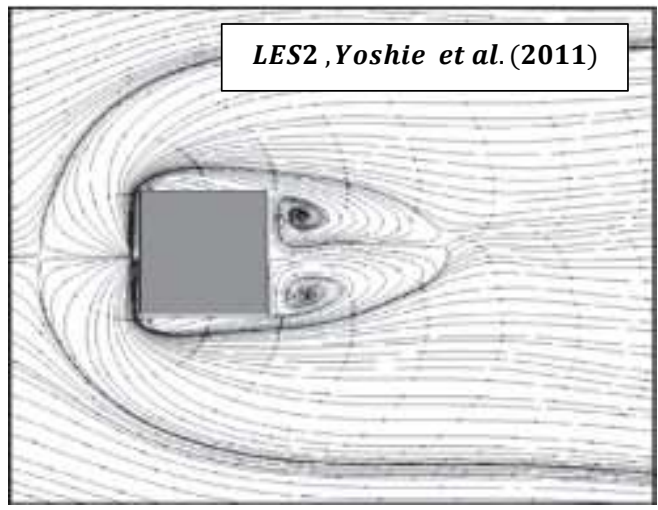

(d)
472

473

474

475

476

477

478

479

480

Figure 12 Horizontal distribution of the streamlines near the ground $\left(\frac{X_{3}}{H}=\mathbf{0 . 0 2 5}\right)$ : (a) experiment by Yoshie et al (2011), (b) default $\boldsymbol{k}-\boldsymbol{\varepsilon}$ closure coefficients, (c) modified $\boldsymbol{k}-\boldsymbol{\varepsilon}$ closure coefficients, (d) LES-2 from (Yoshie et al, 2011)

Contours of the temperature distribution on the same position are also illustrated in Fig. 13. For the case using the default closure coefficients, not only is the level of temperature for the ground surface predicted to be in a higher range than the experiment, but a different temperature pattern is further estimated in the wake region behind the building. For the optimized coefficients, however, the temperature level over the ground surface is closer to the experiment while the temperature distribution behind the building is spread shorter than that of the case with the default coefficients. The 
higher temperature observed in the CFD model around the building surface refers to the implemented adiabatic boundary condition; whereas seemingly the building is not completely isolated from the ground surface in the experiment. Even if considering the uncertainty in the near wall measurement, the CFD results by the modified coefficients are more acceptable than those of the default coefficients.

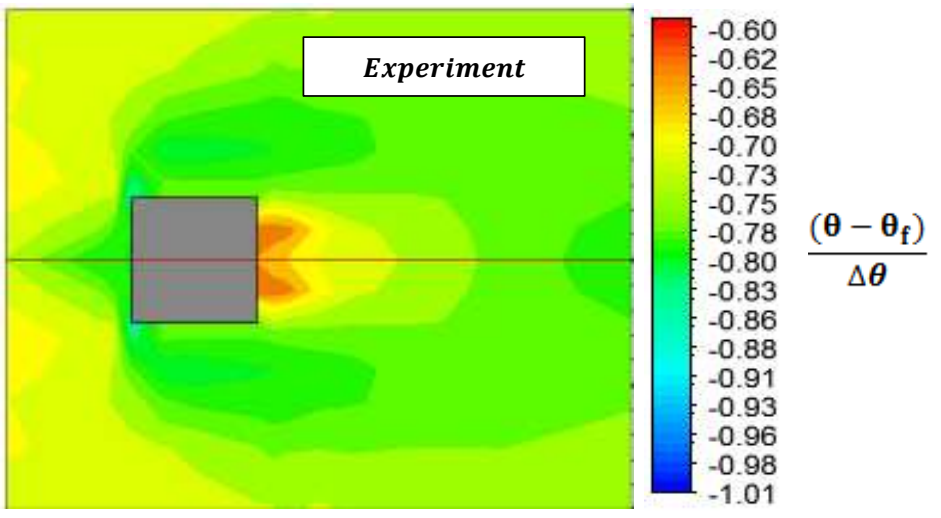

(a)

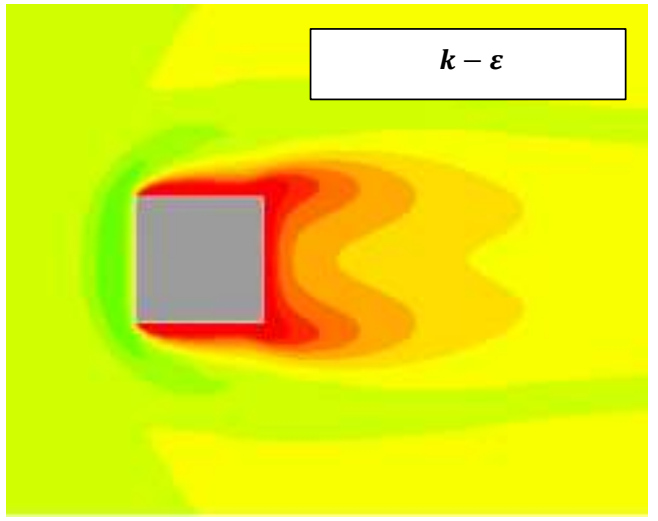

(b)

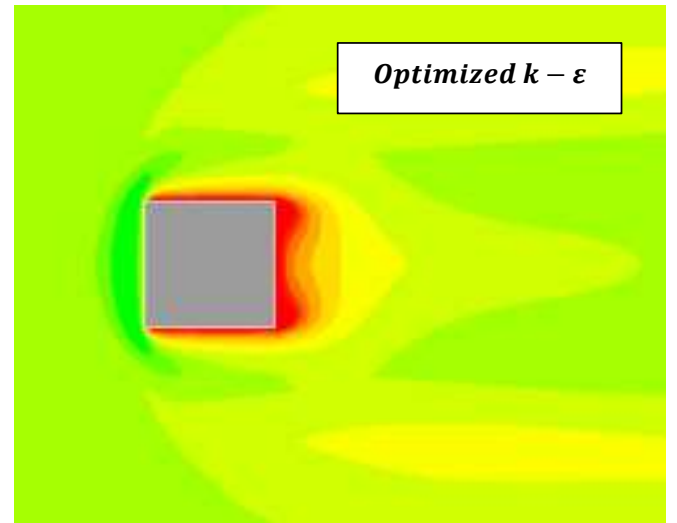

(c)

Figure 13 Contours of the temperature distribution $\frac{\left(\theta-\theta_{\mathrm{f}}\right)}{\Delta \theta}$ near the ground surface: (a) experiment by Yoshie et al (2011), (b) default $\boldsymbol{k}-\boldsymbol{\varepsilon}$ closure coefficients, (c) modified $\boldsymbol{k}-\boldsymbol{\varepsilon}$ closure coefficients

As merely those measurement points that are in the wake region behind the building are considered in the optimization process, it is noteworthy investigating the distribution of flow properties in a high speed region far from the building. In Fig. 14, vertical profiles of the streamwise velocity, turbulent kinetic energy, and temperature along a vertical line placed far from the building at $\frac{\mathrm{x}_{1}}{\mathrm{H}}=2.5$ and $\frac{\mathrm{x}_{2}}{\mathrm{H}}=2$ are shown. Numerical results obtained by the modified coefficient are compared with those obtained by default coefficients and experimental data. Also, results of a LES model presented in (Yoshie et al, 2011) are plotted. It can be seen that the vertical profiles are very similar for the modified and reference cases as well as the experiment. LES model estimated the turbulent kinetic energy more accurately, which refers to the higher accuracy of LES in reproducing the unsteady contribution of turbulent kinetic energy. It is noteworthy to mention that in the current optimization process solely the mean value of the streamwise velocity component was considered; however the accuracy of the modified $k-\varepsilon$ model in predicting the turbulent kinetic energy can be further improved by incorporating the experimental value of $k$ into the optimization process. 


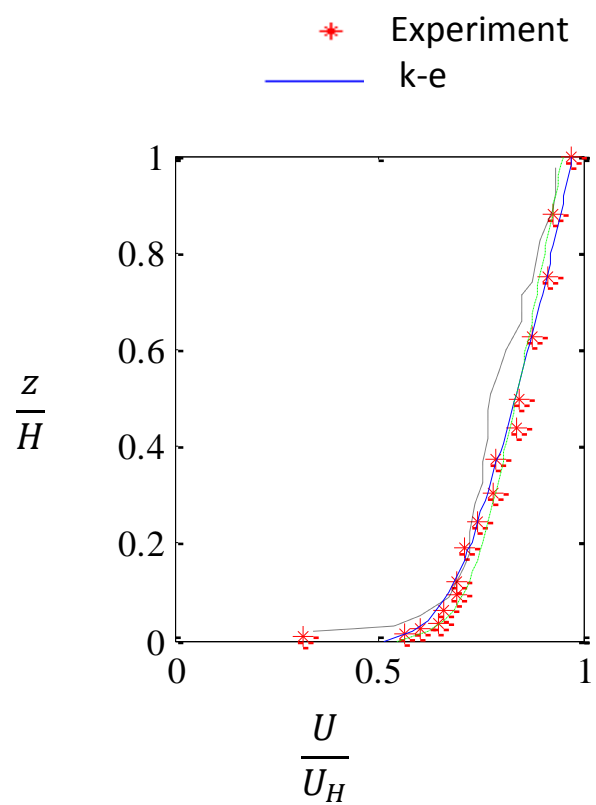

(a)

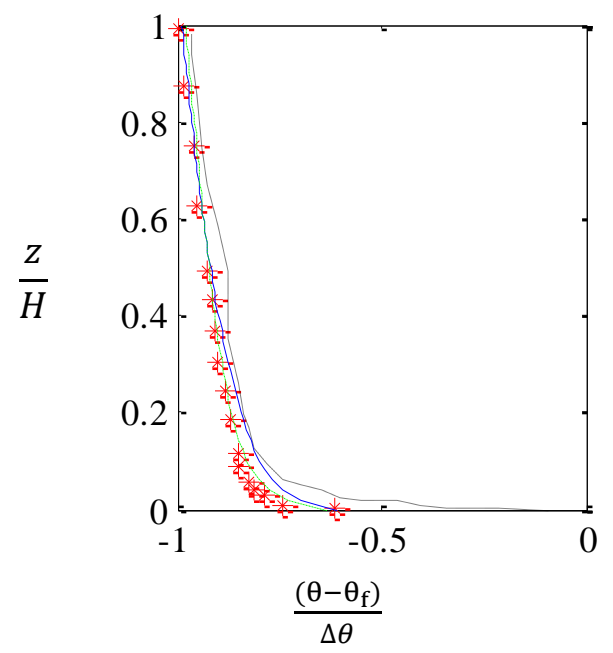

(c)
LES- Yoshie et al. (2011)

Optimized k-e

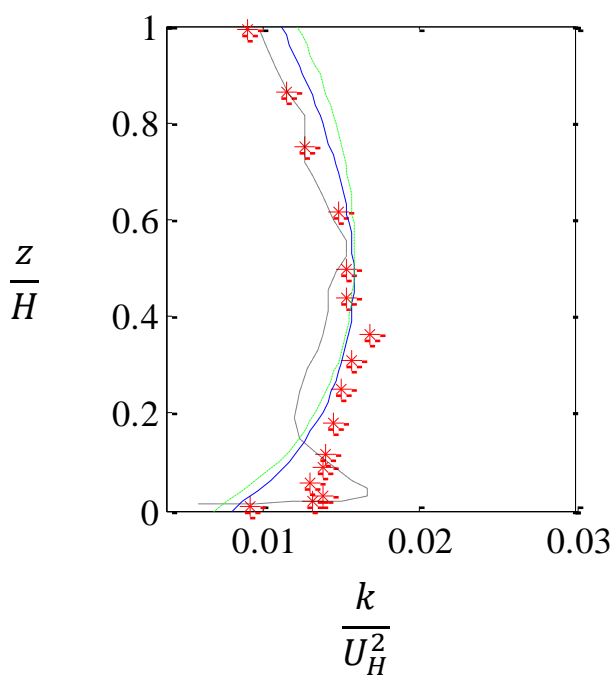

(b)

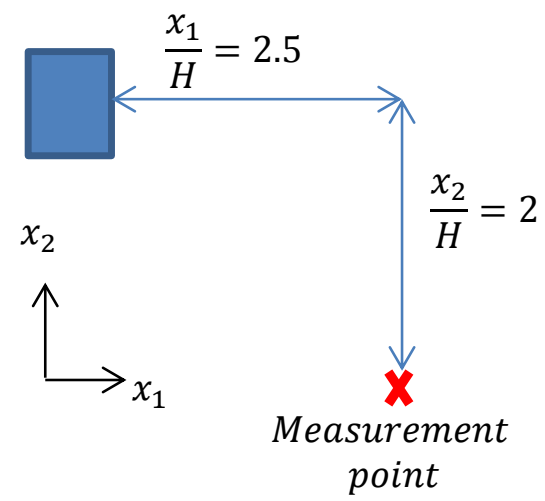

504

Figure 14 Vertical profiles of flow parameters along a vertical line far from the building in high speed region at $\frac{x_{1}}{H}=2.5$ and $\frac{x_{2}}{H}=2$ : (a) streamwise velocity $\frac{U}{U_{H}}$, (b) turbulent kinetic energy $\frac{k}{U_{H}^{2}}$,

(c) non-dimensional temperature $\frac{\left(\boldsymbol{\theta}-\boldsymbol{\theta}_{\boldsymbol{f}}\right)}{\Delta \boldsymbol{\theta}}$, (d) measurement position

\section{Conclusion}

Steady RANS models (including the $k-\varepsilon$ model with Kato-Launder modification) based on the two-equation turbulence models underestimate the momentum diffusion behind the building in the weak wind regions. This results in estimating a large recirculating flow in the wake region and a long reattachment length on the ground. Also, a poor accuracy for the temperature field around the building, specifically in the wake region, is predicted with steady RANS models. Application of the 
default closure coefficients of RANS turbulence models in the popular commercial CFD tools proved to be inaccurate for CFD modeling of the microclimate studies. A systematic approach is therefore proposed in this study in order to improve the accuracy of the RANS family turbulence models applying the stochastic optimization and Monte Carlo Sampling technique. In the optimization process, the closure coefficients were treated as a series of random variables with a given PDF to achieve the best agreement with the experimental data in accordance with the validation metrics. Effectiveness of the proposed methodology for the modification of the closure coefficients of the $k-$ $\varepsilon$ model was shown for simulation scenario of an isolated building placed in a non-isothermal atmospheric boundary layer. In urban areas, because of both the presence of thermal radiation and low air velocity due to the sheltering effect, buoyancy effect is of high importance. A sensitivity analysis was initially conducted to investigate the impact of the $k-\varepsilon$ closure coefficients on the accuracy of the CFD model in comparison with the results of the experimental analysis. The default values of the closure coefficients for the $k-\varepsilon$ model used in the popular CFD tools such as ANSYS CFX, ANSYS FLUENT, PHOENIX and STAR CCM+ are $C_{\mu}=0.09, C_{\varepsilon 1}=1.44, C_{\varepsilon 2}=1.92, \sigma_{k}=1$ and $\sigma_{\varepsilon}=1.3$. However, the recommended values based on the optimization method were found to be $1.45 \leq C_{\varepsilon 1} \leq$ 1.5 and $2.7 \leq C_{\varepsilon 2} \leq 3$ and $0.12 \leq C_{\mu} \leq 0.15$ while the default value of $\sigma_{k}$ was suggested to be acceptable. Based on the numerical results, the modified closure coefficients showed a significant improvement in the accuracy of the CFD model in terms of the velocity, turbulent kinetic energy, and temperature distribution around the building as well as the reattachment length behind the building. The proposed methodology was applied to an isolated building, which is a classical problem in urban aerodynamic, but it can certainly be applied to urban models in dense areas with a group of buildings. Also, it is noteworthy saying, despite the significant improvement in the prediction accuracy achieved by the optimization method, the RANS turbulence models have inherent shortcomings concerning the gradient-diffusion hypothesis and also incapability to reproduce the large-scale fluctuations of flow parameters around the building. Our future work will focus on extending the application of the proposed systematic approach in this study to other CFD modeling examples for the airflow prediction in the urban studies in which we also consider the uncertainty of the turbulent Prandtl number in the energy equation as a calibrating parameter. Through the proposed method, one can find a modified set of the closure coefficients using the available experiment, and then apply the modified coefficients in the CFD model for design and analysis purposes.

\section{Acknowledgement}

The authors would like to express their gratitude to the Guilan Chamber of Commerce and Industries for their financial support.

\section{References}

Allegrini, J., Dorer, V., Carmeliet, J., 2015. Coupled CFD, radiation and building energy model for studying heat fluxes in an urban environment with generic building configurations. Sustainable Cities and Society 19, 385-394.

Capeluto, I.G., Yezioro, A., Shaviv, E., 2003. Climatic aspects in urban design-a case study. Building and Environment 38, 827-835.

CFX, A., 2011. Solver Theory Guide. Ansys. Inc., Canonsburg, PA. 
Cheung, S.H., Oliver, T.A., Prudencio, E.E., Prudhomme, S., Moser, R.D., 2011. Bayesian uncertainty analysis with applications to turbulence modeling. Reliability Engineering \& System Safety 96, 11371149.

Detering, H.W., Etling, D., 1985. Application of the E- $\varepsilon$ turbulence model to the atmospheric boundary layer. Boundary-Layer Meteorology 33, 113-133.

Dunn, M.C., Shotorban, B., Frendi, A., 2011. Uncertainty Quantification of Turbulence Model Coefficients via Latin Hypercube Sampling Method. Journal of Fluids Engineering 133, 041402-041402. Duynkerke, P., 1988. Application of the E- $\varepsilon$ turbulence closure model to the neutral and stable atmospheric boundary layer. Journal of the atmospheric sciences $45,865-880$.

Edeling, W., Cinnella, P., Dwight, R.P., Bijl, H., 2014a. Bayesian estimates of parameter variability in the $k-\varepsilon$ turbulence model. Journal of Computational Physics 258, 73-94.

Edeling, W.N., Cinnella, P., Dwight, R.P., Bijl, H., 2014b. Bayesian estimates of parameter variability in the $k-\varepsilon$ turbulence model. Journal of Computational Physics 258, 73-94.

Evins, R., Allegrini, J., Moonen, P., 2014. Emulating site-specific wind flow information for use in building energy simulations. Building Simulation and Optimization (BSO 2014), London, UK.

Glover, N., Guillas, S., Malki-Epshtein, L., 2011. Statistical calibration of CFD modelling for street canyon flows, Building simulation.

Goerigk, M., Schöbel, A., 2016. Algorithm engineering in robust optimization, Algorithm engineering. Springer, pp. 245-279.

Gousseau, P., Blocken, B., Van Heijst, G., 2011. CFD simulation of pollutant dispersion around isolated buildings: On the role of convective and turbulent mass fluxes in the prediction accuracy. Journal of Hazardous Materials 194, 422-434.

Gousseau, P., Blocken, B., van Heijst, G.J.F., 2013. Quality assessment of Large-Eddy Simulation of wind flow around a high-rise building: Validation and solution verification. Computers \& Fluids 79, 120-133. Guillas, S., Glover, N., Malki-Epshtein, L., 2014. Bayesian calibration of the constants of the turbulence model for a CFD model of street canyon flow. Computer Methods in Applied Mechanics and Engineering 279, 536-553.

Haghighat, F., Mirzaei, P.A., 2011. Impact of non-uniform urban surface temperature on pollution dispersion in urban areas. Building Simulation 4, 227.

Hunt, J., Abell, C., Peterka, J., Woo, H., 1978. Kinematical studies of the flows around free or surfacemounted obstacles; applying topology to flow visualization. Journal of Fluid Mechanics 86, 179-200.

Kastner-Klein, P., Fedorovich, E., Rotach, M.W., 2001. A wind tunnel study of organised and turbulent air motions in urban street canyons. Journal of Wind Engineering and Industrial Aerodynamics 89, 849861.

Kato, M., 1993. The modeling of turbulent flow around stationary and vibrating square cylinders, Ninth Symposium on Turbulent Shear Flows, 1993.

Kato, M., Launder, B., 1993. The modelling of turbulent flow around stationary and vibrating square cylinders. Turbulent Shear Flow 1, 10.14. 11-10.14. 16.

Kim, J., Moin, P., Moser, R., 1987. Turbulence statistics in fully developed channel flow at low Reynolds number. Journal of fluid mechanics 177, 133-166.

Koch, P., Yang, R.-J., Gu, L., 2004. Design for six sigma through robust optimization. Structural and Multidisciplinary Optimization 26, 235-248.

Köse, D.A., Dick, E., 2010. Prediction of the pressure distribution on a cubical building with implicit LES. Journal of Wind Engineering and Industrial Aerodynamics 98, 628-649.

Lakehal, D., Rodi, W., 1997. Calculation of the flow past a surface-mounted cube with two-layer turbulence models. Journal of Wind Engineering and Industrial Aerodynamics 67, 65-78. 
Lateb, M., Meroney, R.N., Yataghene, M., Fellouah, H., Saleh, F., Boufadel, M.C., 2016. On the use of numerical modelling for near-field pollutant dispersion in urban environments - A review. Environmental Pollution 208, Part A, 271-283. Launder, B., Spalding, D., 1974. The numerical computation of turbulent flows. Computer Methods in Applied Mechanics and Energy, 3, 269-289. Magli, S., Lodi, C., Lombroso, L., Muscio, A., Teggi, S., 2015. Analysis of the urban heat island effects on building energy consumption. International Journal of Energy and Environmental Engineering 6, 91-99. Mirzaei, P.A., 2015. Recent challenges in modeling of urban heat island. Sustainable Cities and Society 19, 200-206.

Mirzaei, P.A., Carmeliet, J., 2013. Dynamical computational fluid dynamics modeling of the stochastic wind for application of urban studies. Building and Environment 70, 161-170. Mirzaei, P.A., Haghighat, F., 2010. A novel approach to enhance outdoor air quality: Pedestrian ventilation system. Building and Environment 45, 1582-1593. Mirzaei, P.A., Haghighat, F., 2011. Pollution removal effectiveness of the pedestrian ventilation system. Journal of Wind Engineering and Industrial Aerodynamics 99, 46-58. Mirzaei, P.A., Haghighat, F., 2012. A procedure to quantify the impact of mitigation techniques on the urban ventilation. Building and Environment 47, 410-420. Mirzaei, P.A., Rad, M., 2013. Toward design and fabrication of wind-driven vehicles: Procedure to optimize the threshold of driving forces. Applied Mathematical Modelling 37, 50-61.

Mohamed, M.S., LaRue, J.C., 1990. The decay power law in grid-generated turbulence. Journal of Fluid Mechanics 219, 195-214.

Mori, Y., Hishida, K., Maeda, M., 1995. Buoyancy effects on the wake behind a heated obstacle immersed in a turbulent boundary layer. International journal of heat and fluid flow 16, 405-416.

Murakami, S., 1993. Comparison of various turbulence models applied to a bluff body. Journal of Wind Engineering and Industrial Aerodynamics 46, 21-36.

Murakami, S., 2006. Environmental design of outdoor climate based on CFD. Fluid Dynamics Research 38, 108-126.

Murakami, S., Mochida, A., Hayashi, Y., 1990. examining the $k-\epsilon$ model by means of a wind tunnel test and large-eddy simulation of the turbulence structure around a cube. Journal of Wind Engineering and Industrial Aerodynamics 35, 87-100.

Panofsky, H.A., Dutton, J., 1984. Atmospheric Turbulence: Models and Methods for Engineering Applications, 397 pp. John Wiley, New York.

Pope, S.B., 2001. Turbulent flows. IOP Publishing.

Rhie, C.M., Chow, W.L., 1983. Numerical study of the turbulent flow past an airfoil with trailing edge separation. AIAA Journal 21, 1525-1532.

Richards, P., Mallinson, G., McMillan, D., Li, Y., 2002. Pedestrian level wind speeds in downtown Auckland. Wind and Structures 5, 151-164.

Richards, P., Norris, S., 2011. Appropriate boundary conditions for computational wind engineering models revisited. Journal of Wind Engineering and Industrial Aerodynamics 99, 257-266.

Rodi, W., 1997. Comparison of LES and RANS calculations of the flow around bluff bodies. Journal of Wind Engineering and Industrial Aerodynamics 69, 55-75.

638 Schaefer, J., Hosder, S., West, T., Rumsey, C., Carlson, J.-R., Kleb, W., 2016. Uncertainty quantification 639 of turbulence model closure coefficients for transonic wall-bounded flows. AIAA Journal 55, 195-213.

640 Schittkowski, K., 2006. NLPQLP: A Fortran implementation of a sequential quadratic programming 641 algorithm with distributed and non-monotone line search-user's guide. Report, Department of 642 Computer Science, University of Bayreuth.

643 Shah, H., Hosder, S., Koziel, S., Tesfahunegn, Y.A., Leifsson, L., 2015. Multi-fidelity robust aerodynamic 644 design optimization under mixed uncertainty. Aerospace Science and Technology 45, 17-29. 
Shih, T.-H., Liou, W.W., Shabbir, A., Yang, Z., Zhu, J., 1995. A new k- $\epsilon$ eddy viscosity model for high reynolds number turbulent flows. Computers \& Fluids 24, 227-238.

Shirasawa, T., Mochida, A., Tominaga, Y., Yoshie, R., Kataoka, H., Nozu, T., Yoshino, H., 2003. Development of CFD method for predicting wind environment around a high-rise building, Part2: The cross comparison of CFD results on the flow field around a 4: 4: 1 prism. AlJ Journal of Technology and Design, 441-446.

Solazzo, E., 2008. Modelling dispersion of trafficrelated pollutants in urban canyons and intersections. PhD thesis, University of Birmingham.

Spalart, P., Allmaras, S., 1992. A one-equation turbulence model for aerodynamic flows, 30th Aerospace Sciences Meeting and Exhibit. American Institute of Aeronautics and Astronautics.

Tamura, T., Kawai, H., Kawamoto, S., Nozawa, K., Sakamoto, S., Ohkuma, T., 1997. Numerical prediction of wind loading on buildings and structures-Activities of AIJ cooperative project on CFD. Journal of wind engineering and industrial aerodynamics 67, 671-685.

Tang, Z., Périaux, J., 2012. Uncertainty based robust optimization method for drag minimization problems in aerodynamics. Computer Methods in Applied Mechanics and Engineering 217-220, 12-24. Tari, M., Dahmani, A., 2006. Refined descriptive sampling: A better approach to Monte Carlo simulation. Simulation Modelling Practice and Theory 14, 143-160.

Tavoularis, S., Karnik, U., 1989. Further experiments on the evolution of turbulent stresses and scales in uniformly sheared turbulence. Journal of Fluid Mechanics 204, 457-478.

Todd, A.O., Robert, D.M., 2011. Bayesian uncertainty quantification applied to RANS turbulence models. Journal of Physics: Conference Series 318, 042032.

Tominaga, Y., 2015. Flow around a high-rise building using steady and unsteady RANS CFD: Effect of large-scale fluctuations on the velocity statistics. Journal of Wind Engineering and Industrial Aerodynamics 142, 93-103.

Tominaga, Y., Mochida, A., Shirasawa, T., Yoshie, R., Kataoka, H., Harimoto, K., Nozu, T., 2004. Cross comparisons of CFD results of wind environment at pedestrian level around a high-rise building and within a building complex. Journal of Asian architecture and building engineering 3, 63-70.

Tominaga, Y., Mochida, A., Yoshie, R., Kataoka, H., Nozu, T., Yoshikawa, M., Shirasawa, T., 2008. AlJ guidelines for practical applications of CFD to pedestrian wind environment around buildings. Journal of wind engineering and industrial aerodynamics 96, 1749-1761.

Tominaga, Y., Stathopoulos, T., 2010. Numerical simulation of dispersion around an isolated cubic building: model evaluation of RANS and LES. Building and Environment 45, 2231-2239.

Tsang, C.W., Kwok, K.C.S., Hitchcock, P.A., 2012. Wind tunnel study of pedestrian level wind environment around tall buildings: Effects of building dimensions, separation and podium. Building and Environment 49, 167-181.

Tsuchiya, M., Murakami, S., Mochida, A., Kondo, K., Ishida, Y., 1997. Development of a new k- $\varepsilon$ model for flow and pressure fields around bluff body. Journal of Wind Engineering and Industrial Aerodynamics 67, 169-182.

Van der Velden, A., Koch, P., 2010. Isight design optimization methodologies. ASM Handbook 22.

Vardoulakis, S., Dimitrova, R., Richards, K., Hamlyn, D., Camilleri, G., Weeks, M., Sini, J.-F., Britter, R., Borrego, C., Schatzmann, M., Moussiopoulos, N., 2011. Numerical Model Inter-comparison for Wind Flow and Turbulence Around Single-Block Buildings. Environmental Modeling \& Assessment 16, 169181.

Wong, N.H., Jusuf, S.K., Tan, C.L., 2011. Integrated urban microclimate assessment method as a sustainable urban development and urban design tool. Landscape and Urban Planning 100, 386-389.

Yakhot, V., Orszag, S., Thangam, S., Gatski, T., Speziale, C., 1992. Development of turbulence models for shear flows by a double expansion technique. Physics of Fluids A: Fluid Dynamics (1989-1993) 4, 1510-1520. 
693 Yakhot, V., Orszag, S.A., 1986. Renormalization-group analysis of turbulence. Physical review letters 57, 6941722.

695 Yamada, T., 2004. Merging CFD and atmospheric modeling capabilities to simulate airflows and 696 dispersion in urban areas. Computational Fluid Dynamics Journal 13, 47.

697 Yan, M., Kazuki, H., 1998. Turbulent measurments of the flow field around a high-rise building. Wind 698 Engineers, JAWE 1998, 55-64.

699 Yi, Y.K., Feng, N., 2013. Dynamic integration between building energy simulation (BES) and 700 computational fluid dynamics (CFD) simulation for building exterior surface. Building Simulation 6, 297701308.

702 Yoshie, R., Jiang, G., Shirasawa, T., Chung, J., 2011. CFD simulations of gas dispersion around high-rise 703 building in non-isothermal boundary layer. Journal of Wind Engineering and Industrial Aerodynamics 704 99, 279-288.

705 Yoshie, R., Mochida, A., Tominaga, Y., Kataoka, H., Harimoto, K., Nozu, T., Shirasawa, T., 2007. 706 Cooperative project for CFD prediction of pedestrian wind environment in the Architectural Institute of 707 Japan. Journal of Wind Engineering and Industrial Aerodynamics 95, 1551-1578.

708 Zahid Iqbal, Q.M., Chan, A.L.S., 2016. Pedestrian level wind environment assessment around group of 709 high-rise cross-shaped buildings: Effect of building shape, separation and orientation. Building and 710 Environment 101, 45-63. 